\title{
Interface proliferation and the growth of labyrinths in a reaction-diffusion system
}

\author{
Raymond E. Goldstein* \\ Department of Physics, Joseph Henry Laboratories, Princeton University, Princeton, New Jersey 08544 \\ David J. Muraki ${ }^{\dagger}$ \\ Courant Institute of Mathematical Sciences, New York University, New York, New York 10012 \\ Dean M. Petrich ${ }^{\ddagger}$ \\ Department of Physics, Joseph Henry Laboratories, Princeton University, Princeton, New Jersey 08544 \\ and Department of Physics, California Institute of Technology, Pasadena, California 91125 ${ }^{\S}$
}

(Received 24 July 1995)

\begin{abstract}
In the bistable regime of the FitzHugh-Nagumo model of reaction-diffusion systems, spatially homogeneous patterns may be nonlinearly unstable to the formation of compact "localized states." The formation of space-filling patterns from instabilities of such structures is studied in the context of a nonlocal contour dynamics model for the evolution of boundaries between high and low concentrations of the activator. An earlier heuristic derivation [D. M. Petrich and R. E. Goldstein, Phys. Rev. Lett. 72, 1120 (1994)] is made more systematic by an asymptotic analysis appropriate to the limits of fast inhibition, sharp activator interfaces, and small asymmetry in the bistable minima. The resulting contour dynamics is temporally local, with the normal component of the velocity involving a local contribution linear in the interface curvature and a nonlocal component having the form of a screened Biot-Savart interaction. The amplitude of the nonlocal interaction is set by the activator-inhibitor coupling and controls the "lateral inhibition" responsible for the destabilization of localized structures such as spots and stripes, and the repulsion of nearby interfaces in the later stages of those instabilities. The phenomenology of pattern formation exhibited by the contour dynamics is consistent with that seen by Lee, McCormick, Ouyang, and Swinney [Science 261, 192 (1993)] in experiments on the iodide-ferrocyanide-sulfite reaction in a gel reactor. Extensive numerical studies of the underlying partial differential equations are presented and compared in detail with the contour dynamics. The similarity of these phenomena (and their mathematical description) with those observed in amphiphilic monolayers, type I superconductors in the intermediate state, and magnetic fluids in Hele-Shaw geometry is emphasized.
\end{abstract}

PACS number(s): 82.20.Wt, 03.50.-z, 82.20.Mj, 87.10.+e

\section{INTRODUCTION}

Recent experimental studies $[1,2]$ of pattern formation in reaction-diffusion systems have revealed a mechanism for the generation of space-filling patterns that is markedly different from the classical Turing bifurcation [3]. In the Turing scenario, realized recently in several experiments [4-6], a periodic pattern arises throughout space from a linear instability of a homogeneous state. In contrast, experiments of Lee and co-workers $[1,2]$ have shown that space-filling "labyrinthine" patterns can develop from finite-amplitude perturbations to linearly stable homogeneous states (Fig. 1). These labyrinths are characterized by patches having different chemical compositions that are separated by relatively sharp interfaces, or fronts. The pattern formation process involves the motion of these fronts, which are generally observed to be mutually repelling, thus preventing self-

\footnotetext{
*Electronic address: gold@davinci.princeton.edu

${ }^{\dagger}$ Electronic address: muraki@cims.nyu.edu

${ }^{\ddagger}$ Electronic address: dpetrich@asphodel.caltech.edu

${ }^{\S}$ Present address.
}

crossings and associated changes in topology. The observations that this system possesses bistability, requires finite-amplitude disturbances for nucleation of patterns, and displays fingering instabilities of compact domains suggests a connection to the one-dimensional, reaction-

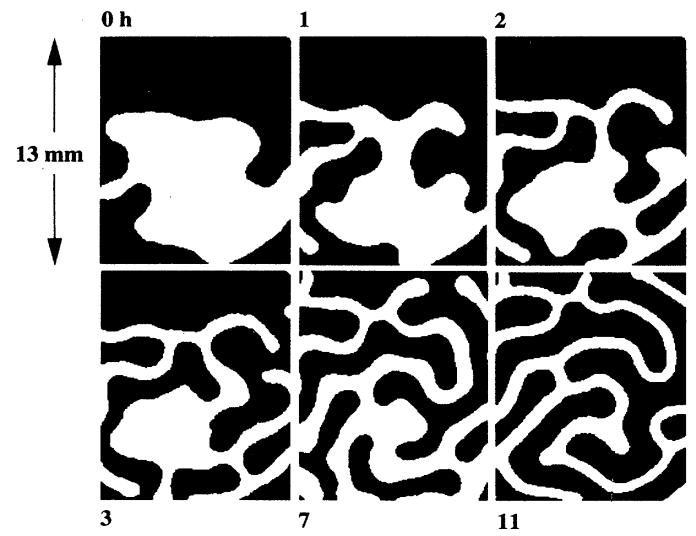

FIG. 1. Chemical pattern formation in the iodide-ferrocyanide-sulfite reaction of Lee et al. [1]. Low and high $\mathrm{pH}$ regions appear, respectively, as white and black by means of a $\mathrm{pH}$ indicator. Times proceed from upper left to lower right in hours following a perturbation. 
diffusion "localized states" considered by Koga and $\mathrm{Ku}$ ramoto [7]. Subsequent generalizations to higher dimensions by Ohta, Mimura, and Kobayashi [8] revealed that these localized states could exhibit fingering instabilities $[9]$.

In earlier work [10] it was suggested on the basis of heuristic arguments that this kind of pattern formation by interacting chemical fronts could be understood by means of a "nonlocal contour dynamics model," derived from the well-known FitzHugh-Nagumo model of activator-inhibitor competition $[11,12]$ in the limit of fast inhibition. This law of motion derives from the combination of a Young-Laplace force associated with interface curvature [13] and a screened Biot-Savart coupling between distant segments of the interface. This nonlocal contribution embodies the phenomenon of "lateral inhibition" - an inhibitory action on a scale much larger than the activator front thickness $[14,15]$. Numerical studies of the contour dynamics model revealed the essential features seen in experiments: the instabilities of compact structures, repulsion of chemical fronts, and the relaxation of branched structures to compact ones as control parameters are varied.

The competition between Young-Laplace and BiotSavart forces has been shown to appear in a variety of other pattern-forming systems, each of which exhibits labyrinthine interface evolution. These include monolayers of dipolar molecules at the air-water interface [16], magnetic fluids [17] in Hele-Shaw flow [18-20], type-I su-

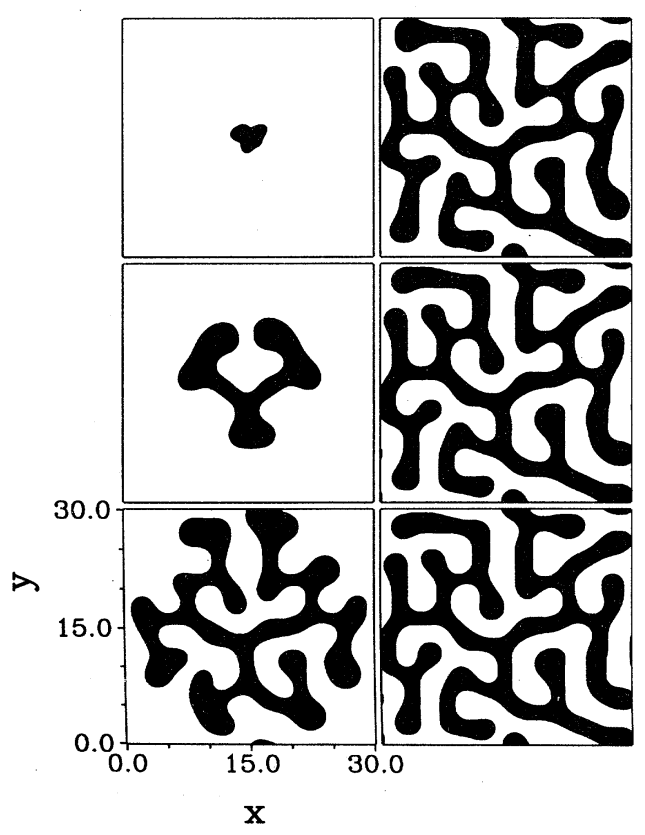

FIG. 2. Simulation of the reaction-diffusion PDEs showing a compact initial condition undergoing a fingering instability, eventually producing a space-filling labyrinth. Panels are contour plots with $u \leq 0.5$ shown white and $u \geq 0.5$ shown black, at rescaled times of $\tau=0,5,10,15,20,25$. The parameters in Eq. (2.4) are $D=0.01, \rho=0.15$, and $r=0.52$; thus, the state $u=1$ (black) is less stable than $u=0$ (white). perconductors in the intermediate state [21], and thin garnet films [22]. In each of these cases, the patterns are defined by the boundaries between two thermodynamic phases in coexistence, with bulk electric or magnetic dipolar order. The Biot-Savart interactions then arise from the usual correspondence between magnetization and current loops. The common phenomenology of these systems has been reviewed recently [23].

Here we elaborate on the contour dynamics in two ways: a systematic derivation is presented using matched asymptotics, and extensive numerical evidence is produced to confirm consistency with the phenomenology of the original reaction-diffusion partial differential equations (PDEs). Throughout the analysis we emphasize the variational structure of the dynamics, both in the fastinhibitor limit and more generally, for it appears not to be widely appreciated that complex patterns can arise from purely gradient flows.

In order to motivate the major emphases in this work, we show in Figs. 2 and 3 two numerical simulations of the pattern formation exhibited by the FitzHugh-Nagumo PDE model. The regions in which the activator takes on each of its bistable values are indicated by black and white. In Fig. 2 we see a compact initial domain of one phase expand and finger to produce a space-filling labyrinthine pattern. Yet, a small change in parameter values leads to the relaxation of this pattern back to a stable localized state, as shown in Fig. 3. In this paper we show that the complex pattern formation observed in Figs. 2 and 3 can, in fact, be explained in terms of three elementary processes: (1) stripe stabilization and

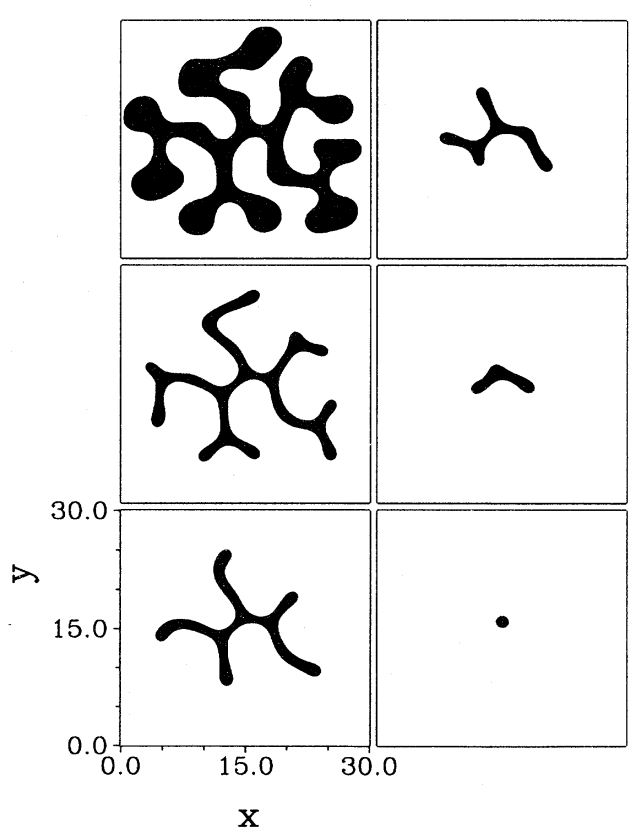

FIG. 3. Reaction-diffusion simulations as in Fig. 2, but starting with a branched domain, and with $D=0.01$, $\rho=0.10$, and $r=0.60$. With these parameter values, a circular localized state is the stable configuration to which the system relaxes. 
repulsion; (2) domain localization; (3) interface proliferation and transverse front instabilities. The remainder of the paper is organized around the elucidation of each of these features on both energetic grounds and by means of asymptotic methods for the derivation of front motion from PDEs. The particular reaction-diffusion model of interest here is introduced in Sec. II along with a discussion of its stability and variational structure. We illustrate the region in parameter space where the Turing instability is precluded and localized states appear and discuss the gradient-flow nature of the dynamics in the fast-inhibitor limit. Interface dynamics in this limit is considered in detail in Secs. III and IV, where we reiterate the heuristic arguments leading to front dynamics and present the asymptotic analysis. Section III focuses on one-dimensional systems where the features of stripe stabilization (a form of domain localization) and stripe repulsion are most easily understood. Two-dimensional systems are considered in Sec. IV, in which a variant on domain localization is found. A detailed discussion of transverse front instabilities is given in Sec. V, revealing a common mechanism not unlike that of the MullinsSekerka instability in solidification. The numerical studies of both the full PDEs and the contour dynamics are presented in Sec. VI. Some considerations regarding the form of the dynamics away from the fast-inhibitor limit are outlined in Sec. VII. Nonlocal interface dynamics in the various contexts described above are tied together in Sec. VIII, and Sec. IX outlines our conclusions and open problems. For completeness, an Appendix details the numerical methods used for the study of both the PDEs and the contour dynamics.

There have been several other recent theoretical studies relevant to the experiments of Lee et al. [1,2]. In extensive simulations of the Gray-Scott model [24], Pearson [25] has found a wide variety of patterns, including ordered arrays of spots, lamellar stripe domains, and labyrinthine structures. Some of these were found to arise from finite-amplitude perturbations, as in the experimental work. More recently, Hagberg and Meron $[26,27]$, based on reaction-diffusion equations similar to the FitzHugh-Nagumo model, have provided elegant geometrical arguments for a connection between labyrinthine instabilities in the fast-inhibitor limit and spiral wave behavior for slow inhibition. The present work is complementary to these in providing a more detailed picture of the fast-inhibitor limit, where the dynamics reduces to a pure gradient flow. Clearly, however, certain phenomena are precluded by the fast-inhibitor limit, such as spot "self-replication" studied in recent experimental [28] and theoretical works $[25,29,30]$.

\section{THE MODEL}

\section{A. Definition}

Our starting point is the FitzHugh-Nagumo model [11] for the coupled dynamics of an activator $u$ and inhibitor $v$. We choose appropriate definitions of $u, v$, space, and time to arrive at the nondimensionalized form

$$
\begin{gathered}
u_{t}=D \nabla^{2} u-F^{\prime}(u ; r)-\rho(v-u), \\
\epsilon v_{t}=\nabla^{2} v-v+u
\end{gathered}
$$

Here, $D$ is the activator diffusion constant normalized to that of the inhibitor and $F(u ; r)$ is a double-well potential representing the autocatalytic behavior of the activator. In a convenient parametrization whereby the two local minima in $F$ are at $u=0$ and $u=1$, the potential $F(u ; r)$ is written in terms of a single symmetry-breaking control parameter $r$ as

$$
F(u ; r)=\frac{1}{4} u^{2}(u-1)^{2}+\left(r-\frac{1}{2}\right)\left(\frac{1}{2} u^{2}-\frac{1}{3} u^{3}-\frac{1}{12}\right)
$$

provided $0<r<1$. When $r$ differs from $\frac{1}{2}$, a difference in potential is created between the two states,

$$
\begin{aligned}
\Delta F & \equiv F(u=1 ; r)-F(u=0 ; r) \\
& =\frac{1}{6}\left(r-\frac{1}{2}\right)
\end{aligned}
$$

so that for $\Delta F>0\left(r>\frac{1}{2}\right)$ the state $u=0$ is the more stable, and the reverse for $\Delta F<0$. In Figs. 2 and 3 , the $u=0$ state is associated with the white area and $u=1$ with black.

With the potential $F$ as defined above, the model PDE is a coupled diffusion system which is nonlinear only in the activator equation

$$
\begin{gathered}
u_{t}=D \nabla^{2} u-u(u-r)(u-1)-\rho(v-u), \\
\epsilon v_{t}=\nabla^{2} v-(v-u) .
\end{gathered}
$$

This particular nondimensional formulation has the advantage of possessing an exact invariance under the simultaneous transformations

$$
u \rightarrow 1-u ; \quad v \rightarrow 1-v ; \quad r \rightarrow 1-r .
$$

This property implies that we need not consider as distinct cases the evolution of black spots in white domains versus white spots in black domains. Within the context of the PDE model (2.4) we therefore focus on the following question: "By what mechanism do black labyrinthine patterns develop in a white domain?" The form of the inhibitor dynamics embodies both its self-limiting behavior and its stimulation by the activator, while the linear coupling to $v$ in the activator dynamics is the simplest such term. Although it is traditional to view $v$ itself as the inhibitory agent, we have chosen instead to introduce an inhibitor coupling in the form $\rho(v-u)$ which insures that the state $u=v=1$ remains a stationary uniform state for all $\rho$.

The parameter $\epsilon$ in Eq. (2.1b) distinguishes between the slow-inhibitor limit $(\epsilon \gg 1)$ and the fast-inhibitor regime $(\epsilon \ll 1)$. We assume the latter, a limit that is opposite to the limit assumed in phase-field models [31] and spiral wave dynamics [32]. Through the nondimensionalizations used in (2.4), the natural diffusive length scale of the inhibitor is set to unity. The corresponding length scale for the activator is $\sqrt{D}$, so the limit of sharp 
activator fronts of interest here requires $\sqrt{D} \ll 1$. The parameter $D$ serves to mainly to set the width of those fronts, while the two remaining parameters in the model, $r$ and $\rho$, will be seen to control more global aspects of the front dynamics.

\section{B. Stability against the Turing bifurcation}

A simple stability analysis shows that both states $u=$ 0 and $u=1$ can be linearly stable simultaneously to all periodic disturbances. Consider first the state $u=$ $v=0$. In the limit of fast inhibition, $\epsilon \rightarrow 0$ and for $0<r<1$ (so that both minima of $F$ exist), one finds that perturbations having the form

$$
\begin{aligned}
& u=U e^{i k x+\sigma t}, \\
& v=V e^{i k x+\sigma t}
\end{aligned}
$$

are characterized by two branches of solutions for the growth rates $\sigma$,

$$
\begin{aligned}
& \sigma_{+}=-D k^{2}-r+\rho \frac{k^{2}}{1+k^{2}}+O(\epsilon), \\
& \sigma_{-}=-\frac{1+k^{2}}{\epsilon}+O(1) .
\end{aligned}
$$

While the branch $\sigma_{-}$is clearly damped for all wave vectors, $\sigma_{+}$may become positive for $\rho$ exceeding a critical value $\rho_{c}(r)$. The neutral curve for this linear (Turing) instability, as defined by the conditions $\sigma_{+}(k)=0$ and $d \sigma_{+}(k) / d k=0$, is

$$
\rho_{T}(r) \sim(\sqrt{r}+\sqrt{D})^{2}
$$

so that when $\rho<\rho_{T}(r)$ the state $u=v=0$ is linearly stable. At the critical $\rho=\rho_{T}(r)$ the marginally stable wave vector $k_{T}$ is

$$
k_{T} \sim\left(\sqrt{\frac{\rho_{T}}{D}}-1\right)^{1 / 2}=\left(\frac{r}{D}\right)^{1 / 4}
$$

Appealing to the symmetry relations in Eq. (2.5), we deduce that the stability criterion for the state $u=v=1$ is $\rho<\rho_{T}(1-r)$, where the function $\rho_{T}$ is the same as in (2.8). Thus, simultaneous stability of both homogeneous states to the Turing bifurcation requires $\rho<\rho_{T}(r)$ and $\rho<\rho_{T}(1-r)$. The region in $\rho-r$ space so defined is shown in Fig. 4 for the case $D=0.01$. The inset of Fig. 4 shows two curves of $\sigma_{+}(k)$ for the parameters $(r=0.65, \rho=0.25)$ (stable) and $(r=0.65, \rho=0.60)$ (unstable).

As the markers on Fig. 4 suggest, the labyrinthine dynamics of Figs. 2 and 3 are observed near $\rho \approx 0$ and $r \approx \frac{1}{2}-$ values that are well within the stable regime. Although for these parameters the system is stable against the Turing mechanism, it is the underlying bistability that permits the existence of nontrivial localized states through intrinsically nonlinear processes.

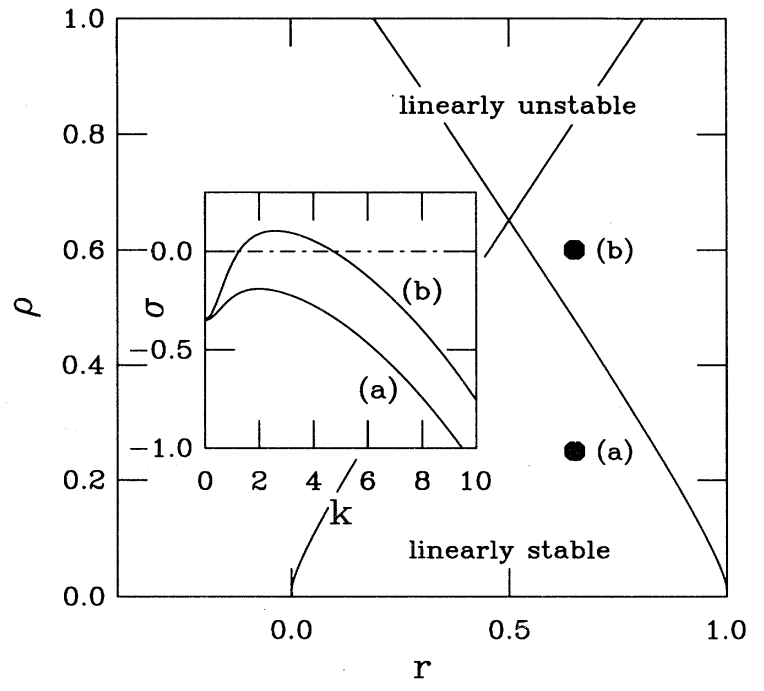

FIG. 4. Regions of the $r-\rho$ parameter space in which Turing bifurcations are possible (above solid lines) and forbidden (below) within linear stability. Inset shows growth rate of the most dangerous mode as a function of wave number in both the linearly stable and unstable regions, for values indicated by the symbols.

\section{Variational aspects}

Equations (2.4) are neither purely dissipative nor Hamiltonian, but can be expressed in a variational form

$$
\begin{aligned}
& u_{t}=-\frac{\delta \mathcal{E}_{u}}{\delta u}-\rho \frac{\delta \mathcal{F}}{\delta u} \\
& \epsilon v_{t}=-\frac{\delta \mathcal{E}_{v}}{\delta v}+\frac{\delta \mathcal{F}}{\delta v},
\end{aligned}
$$

where the energy functionals are

$$
\begin{aligned}
\mathcal{E}_{u} & =\int d \mathbf{x}\left\{\frac{1}{2} D|\nabla u|^{2}+F(u ; r)-\frac{1}{2} \rho u^{2}\right\}, \\
\mathcal{E}_{v} & =\int d \mathbf{x}\left\{\frac{1}{2}|\nabla v|^{2}+\frac{1}{2} v^{2}\right\}, \\
\mathcal{F} & =\int d \mathbf{x} u v
\end{aligned}
$$

It is precisely because the cross terms in the dynamics (2.4) are of opposite sign that the system is not a pure gradient flow for finite $\epsilon$. One may verify that neither $\mathcal{E}_{u}$, nor $\mathcal{E}_{v}$, nor $\mathcal{F}$ decreases monotonically in time. This completely changes in the fast-inhibitor limit [33] $(\epsilon \rightarrow$ $0)$, for then Eq. (2.10b) becomes a functional relation

$$
\frac{\delta \mathcal{E}_{v}}{\delta v}=\frac{\delta \mathcal{F}}{\delta v}
$$

from which it follows that the combination $\mathcal{E}_{u}+\rho \mathcal{F}$ decreases monotonically in time 


$$
\frac{\partial}{\partial t}\left(\mathcal{E}_{u}+\rho \mathcal{F}\right)=-\int d^{2} x\left(\frac{\delta \mathcal{E}}{\delta u}+\rho \frac{\delta \mathcal{F}}{\delta u}\right)^{2} .
$$

The dynamics is then a gradient flow, a feature central to the heuristic arguments given in earlier work [10] and elaborated on below.

\section{The nonlocal energy functional}

With the exception of Sec. VII, for the remainder of this paper we shall assume the fast-inhibitor limit $\epsilon=0$. The inhibitor dynamics degenerates into an instantaneous-in-time relation between $v$ and $u$ given by Eq. (2.12). Using the specific forms of $\mathcal{E}_{v}$ and $\mathcal{F}$, it reduces to

$$
\left(\nabla^{2}-1\right) v(\mathbf{x}, t)=-u(\mathbf{x}, t)
$$

which can be solved using a Green's function

$$
v(\mathbf{x}, t)=\int d \mathbf{x}^{\prime} \mathcal{G}\left(\mathbf{x}-\mathbf{x}^{\prime}\right) u\left(\mathbf{x}^{\prime}\right)
$$

In one and two dimensions, respectively, the Green's functions are

$$
\begin{gathered}
\mathcal{G}\left(x-x^{\prime}\right)=\frac{1}{2} e^{-\left|x-x^{\prime}\right|}, \\
\mathcal{G}\left(\mathbf{x}-\mathbf{x}^{\prime}\right)=\frac{1}{2 \pi} K_{0}\left(\left|\mathbf{x}-\mathbf{x}^{\prime}\right|\right),
\end{gathered}
$$

where $K_{0}$ is the modified Bessel function of order zero.

Substituting for $v$ in Eq. (2.4a), we obtain the spatially nonlocal activator PDE

$u_{t}=D \nabla^{2} u-F^{\prime}(u ; r)+\rho u-\rho \int d \mathbf{x}^{\prime} \mathcal{G}\left(\mathbf{x}-\mathbf{x}^{\prime}\right) u\left(\mathbf{x}^{\prime}\right)$.

From the arguments leading up to Eq. (2.13), and as had been observed earlier by Ohta, Ito, and Tetsuka [34], Eq. (2.17) has the variational form $u_{t}=-\delta \mathcal{E} / \delta u$ with the energy functional

$$
\begin{aligned}
\mathcal{E}[u]= & \int d \mathbf{x}\left\{\frac{1}{2} D|\nabla u|^{2}+F(u ; r)-\frac{1}{2} \rho u^{2}\right\} \\
& +\frac{1}{2} \rho \int d \mathbf{x} \int d \mathbf{x}^{\prime} u(\mathbf{x}) \mathcal{G}\left(\mathbf{x}-\mathbf{x}^{\prime}\right) u\left(\mathbf{x}^{\prime}\right) .
\end{aligned}
$$

While, as remarked earlier, $\mathcal{E}$ decreases monotonically in time, $\mathcal{E}$ is not necessarily bounded from below in an infinite domain, and this variational principle does not guarantee a stationary long-time limit.

As an aside, if the variations in $u$ are on length scales long compared to the $O(1)$ inhibitor screening length, then we can expand the integrand in (2.18) for $\mathbf{x}^{\prime}$ near $\mathbf{x}$ and obtain up to fourth order the gradient expansion

$$
\iint u \mathcal{G} u \simeq \int d \mathbf{x}\left(u^{2}-|\nabla u|^{2}+\left(\nabla^{2} u\right)^{2}+\cdots\right) .
$$

Collecting together terms, we obtain an approximate local theory,

$$
\mathcal{E} \simeq \int d \mathbf{x}\left\{\frac{1}{2} \bar{D}|\nabla u|^{2}+\frac{1}{2} \rho\left(\nabla^{2} u\right)^{2}+F(u ; r)\right\}
$$

where $\bar{D}=D-\rho$. At this level, the energy functional is very similar to that which appears in the SwiftHohenberg model [35], as well as the theory of Lifshitz points in condensed matter systems [36], and leads quite naturally to modulated patterns by virtue of the possibly negative coefficient of $|\nabla u|^{2}$. We note however, that such a gradient expansion is only of limited utility in the limit of sharp interfaces of interest in the present work. Nevertheless, an approximate local theory can be developed within the limit of sharp interfaces when the curvature of the interface is small, as shown in Sec. VB.

Let us note, finally, one important feature of Eq. (2.20). We see that the lowest-order term in the gradient expansion (2.19) leads to a contribution $(1 / 2) \rho u^{2}$ in the energy functional. This precisely cancels the term $-(1 / 2) \rho u^{2}$ seen in Eq. (2.18), which arises from the $-\rho(v-u)$ inhibition coupling. Thus, the effective potential in a local theory (2.20) is precisely the function $F(u ; r)$, a convenient simplification in subsequent sections.

\section{INTERFACE MOTION IN ONE DIMENSION}

\section{A. Energetic derivation of a stripe evolution}

In the PDE dynamics shown in Figs. 2 and 3 , the potential difference $\Delta F$ is positive so that $u=0$ (white) is the energetically preferred state. Nonetheless, in both cases the black domain survives despite its energetic disadvantage. Insight into this bistable configuration can be obtained through an analysis of a single black stripe.

Consider the dynamics of an interval with $u \approx 1$ in a background of $u \approx 0$ (i.e., the cross section of a black stripe). A PDE computation of (2.4) for one such evolution is shown in Fig. 5, where we see an approach of the two sharp activator $(u)$ fronts that mark the transition from black to white. In contrast, the inhibitor field $v$ is considerably more diffuse. But perhaps the most notable aspect about this particular evolution is that the black stripe asymptotically stabilizes at a finite size. By applying a simple heuristic argument that was outlined in an earlier work [10], an approximate dynamics for this process can be derived that predicts the equilibrium width of this black localized state.

The argument begins from the essential assumption that the activator fronts are narrow relative to the scale of the pattern, so that $u$ may be taken to be piecewise constant away from fronts. We focus on the case of a single black stripe of $u=1$ located symmetrically between $x=-Q(t)$ and $x=+Q(t)$ in an otherwise quiescent white background $(u=0)$. The evolution of $Q(t)$ is deduced from the gradient dynamics based on the onedimensional version of the energy functional (2.18) 


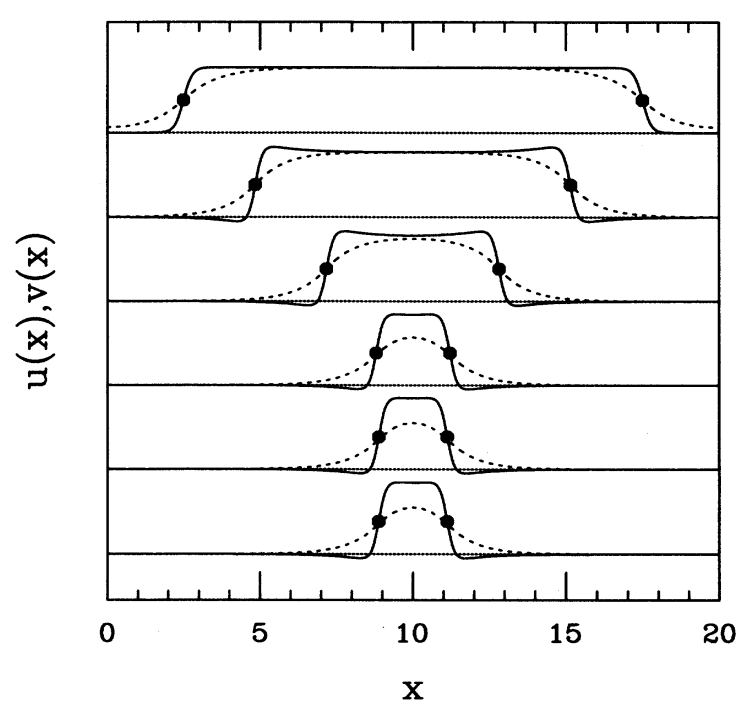

FIG. 5. Space-time portrait of the formation of a localized state in one dimension by the shrinkage of a region of $u=1$. Activator is shown as a solid line, inhibitor as a dashed line, with time increasing downwards. Solid symbols locate the value $u=\frac{1}{2}$. Parameter values are $D=0.01, r=0.55$, $\rho=0.15$.

$$
\begin{aligned}
\mathcal{E}[u]= & \int_{-\infty}^{\infty} d x\left\{\frac{1}{2} D u_{x}^{2}+F(u ; r)-\frac{1}{2} \rho u^{2}\right\} \\
& +\frac{1}{2} \rho \int_{-\infty}^{\infty} d x \int_{-\infty}^{\infty} d x^{\prime} u(x) \mathcal{G}\left(x-x^{\prime}\right) u\left(x^{\prime}\right)
\end{aligned}
$$

To insure a finite energy, we consider the energy difference $\Delta \mathcal{E}=\mathcal{E}[u]-\mathcal{E}\left[u_{\infty}\right]$, where $u_{\infty}$ is the value $u$ takes as $x \rightarrow \pm \infty$. Then, in following the prescription of the earlier results [10], the energy functional for a stripe is partitioned into three contributions: an effective line tension $\gamma$, a pressure $\Pi$, and a nonlocal coupling between fronts. These contributions are explicitly calculated below.

In calculating $\Delta \mathcal{E}$ for the stripe, the line tension consists of the contributions that are localized at the fronts (like $u_{x}^{2}$ ). In one dimension, this requires a contribution to $\Delta \mathcal{E}$ that is some constant $\gamma$ proportional to the number of fronts. On dimensional grounds, the line tension associated with a single front $\gamma$ can be estimated by the gradient integral

$$
\gamma \approx \int_{\text {front }} d x D u_{x}^{2}=O\left(\frac{D}{\ell}\right)
$$

where $\ell$ is a characteristic length scale of the front. Since this length scale must be the diffusive length $\sqrt{D}$, we expect $\gamma \sim \sqrt{D}$.

Under the assumption that $u$ is piecewise constant and either zero or unity, the evaluation of the pressure and nonlocal contributions to the energy functional are considerably simplified. By virtue of having subtracted the white background energy $\mathcal{E}\left[u_{\infty}\right]$, the contribution arising from the potential $F$ is restricted to the stripe, and is thus proportional to the pulse length $2 Q$. The constant of proportionality associated with the integral of the potential function $F$ is interpreted as a pressure $\Pi$ and is given by

$$
\Pi \equiv \frac{1}{2 Q} \int_{-Q}^{+Q} d x\{F(1 ; r)-F(0 ; r)\}=\Delta F
$$

The remaining two terms in the energy integral (3.1) are proportional to the inhibition coupling $\rho$ and are also integrations only over domains where $u=1$. For the single black stripe, these two integrals, when combined, require the evaluation of

$$
-\frac{1}{2} \rho \int_{-Q}^{Q} d x\left\{1-\int_{-Q}^{Q} d x^{\prime} \mathcal{G}\left(x-x^{\prime}\right)\right\}
$$

Using the PDE for the Green's function $\mathcal{G}=\mathcal{G}_{x x}+\delta(x-$ $\left.x^{\prime}\right)$ and the reciprocity relation $\mathcal{G}_{x x}=-\mathcal{G}_{x x^{\prime}}$, the integral (3.4) simplifies to

$$
-\frac{1}{2} \rho \int_{-Q}^{Q} d x \int_{-Q}^{Q} d x^{\prime} \mathcal{G}_{x x^{\prime}}=\rho[\mathcal{G}(2 Q)-\mathcal{G}(0)] .
$$

Combining all three contributions $(3.2,3.3,3.5)$ the energy of a localized state of size $2 Q$ is thus estimated to be

$$
\Delta \mathcal{E}[Q] \simeq 2 \gamma+2 Q \Pi+\rho[\mathcal{G}(2 Q)-\mathcal{G}(0)]
$$

As remarked in the paragraph following Eq. (2.20), we see that the pressure contribution $\Pi$ comes from the bare function $F(u ; r)$, rather than from the shifted function $F-(1 / 2) \rho u^{2}$ in Eq. (2.18). Furthermore, the line tension constant $\gamma$ as estimated from Eq. (3.2) is in fact asymptotically correct, despite an apparent discrepancy of a factor of two between (3.1) and (3.2). This detail is resolved by the asymptotic analysis in the following section, and involves the recognition of an additional $\mathcal{O}(\sqrt{D})$ contribution from a boundary correction to the estimate in (3.3).

The final step in this heuristic derivation is the translation of the variational principle $u_{t}=-\delta \mathcal{E} / \delta u$ into an equation of motion for the front position $Q(t)$. In terms of the general theory of Lagrangian dynamics, viscous forces can be introduced as the variational of a Rayleigh dissipation functional $\mathcal{R}[37]$,

$$
\mathcal{R}\left[u_{t}\right] \equiv \frac{1}{2} \int_{-\infty}^{+\infty} d x u_{t}^{2}
$$

so that the dynamics can be written in the variational form

$$
\frac{d}{d t} \frac{\partial \mathcal{L}}{\partial u_{t}}-\frac{\partial \mathcal{L}}{\partial u}=-\frac{\partial \mathcal{R}}{\partial u_{t}}
$$

For a single moving front having an invariant spatial profile, the dissipation functional is intimately related to the line tension constant $\gamma$ since 


$$
\begin{aligned}
\mathcal{R}\left[u_{t}\right] & \equiv Q_{t}^{2} \int_{\text {front }} d x u_{x}^{2} \\
& \approx \frac{\gamma}{D} Q_{t}^{2}
\end{aligned}
$$

where the result is doubled to account for both fronts. Now, adopting $Q$ as the dynamical coordinate and taking for the Lagrangian the energy difference $\mathcal{L}[Q]=-\Delta \mathcal{E}[Q]$, the variational principle (3.8) gives a dynamical equation for the front location $Q(t)$

$$
Q_{t}=-\frac{D}{2 \gamma} \frac{\partial \Delta \mathcal{E}}{\partial Q}=-\frac{D}{\gamma}\left[\Delta F-\frac{\rho}{2} \mathrm{e}^{-2 Q}\right] .
$$

Note that the absence of any $u_{t}$ dependence in the Lagrangian $\mathcal{L}[u]$ leads to an inertialess front dynamics. The possibility of inertial contributions to the front evolution is discussed in Sec. VII.

We deduce from (3.10) that black localized states are only possible if $\Delta F>0$, so that the state $u=1$ has the higher energy of the two minima of $F(u)$. Moreover, beyond the threshold $\rho=2 \Delta F$ the inhibitor coupling supports a stable black stripe of width

$$
2 Q^{*} \sim \ln \left(\frac{3 \tilde{\rho}}{\tilde{r}}\right)
$$

and whose associated energy is

$$
\frac{2 \Delta \mathcal{E}}{\sqrt{D}}=\frac{\sqrt{2}}{3}-\tilde{\rho}\left\{1-\frac{\tilde{r}}{3 \tilde{\rho}}\left[1-\ln \left(\frac{\tilde{r}}{3 \tilde{\rho}}\right)\right]\right\} .
$$

We have introduced the scaled parameters

$$
\tilde{r}=\frac{6 \Delta F}{\sqrt{D}}=\frac{r-1 / 2}{\sqrt{D}}, \quad \tilde{\rho}=\frac{\rho}{\sqrt{D}},
$$

which in the following section are shown to follow naturally from an asymptotic analysis. Below threshold, the inhibition coupling is insufficient to prevent annihilation through the collision of two fronts. On the other hand, for $\Delta F<0$, no equilibrium width exists and the stripe expands indefinitely to fill space. The existence of an equilibrium stripe width for the less-preferred state demonstrates, within the context of this PDE model, the process of stripe stabilization.

Using the symmetry relations (2.5), we deduce the converse result that white localized states $(u=0)$ in a black background $(u=1)$ may exist only when $\Delta F<0$. Likewise, a white stripe will expand unboundedly when $\Delta F>0$.

Localized states may also be formed by the nucleation of a small domain of $u=1$, followed by its growth to the stable size. From the form of the energy (3.6) we see that there is a barrier to the creation of a localized state, so this process requires a nucleus larger than some critical size. This is illustrated by the PDE computation in Fig. 6, where we see the growth of a slightly supercritical nucleus.

While we have emphasized the somewhat counterintuitive result that a localized one-dimensional state consists of the phase that has a higher value of the potential $F$,

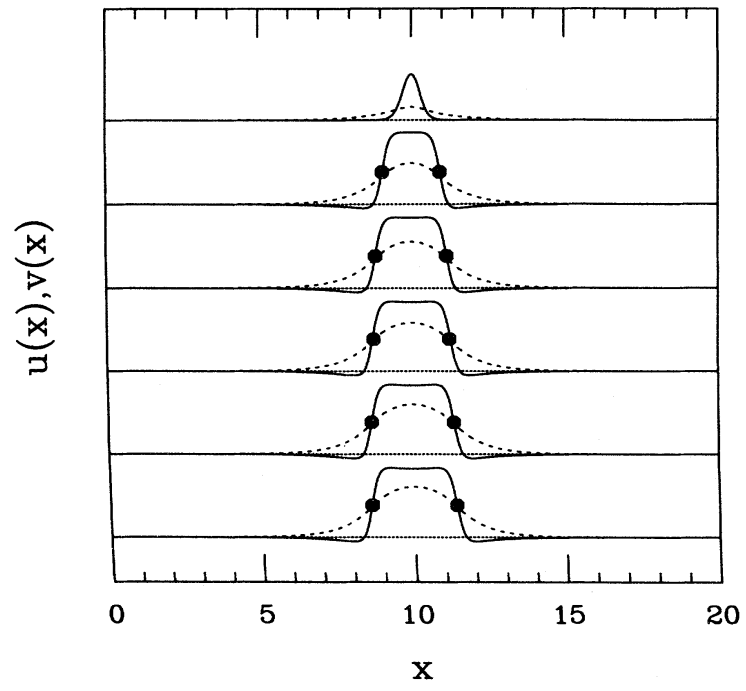

FIG. 6. Space-time portrait of the growth of a localized state in one dimension from a small nucleated domain. Activator is shown as a solid line, inhibitor as a dashed line, with time increasing downwards. Parameters are $D=0.01$, $r=0.52, \rho=0.15$.

it is interesting to note from (3.12) that the total "structural energy" $\Delta \mathcal{E}$ of the stripe can be of either sign. It will ultimately be shown (Sec. V) that the existence of stripes with $\Delta \mathcal{E}=0$ is intimately connected with the onset of the labyrinthine instability.

\section{B. Asymptotic derivation of a front dynamics}

The preceding section describes the dynamics and interaction of interfacial fronts as a Lagrangian relaxation process which is independent of the detailed fine structure of the activator $(u)$ field. Using formal matchedasymptotic arguments $[13,31,32,38]$, these results are substantiated as the leading order behavior of the PDE model (2.1) in a particular limit of weak activator diffusion.

The asymptotic analysis relies on a strong separation of scales between the spatial structures of the activator and inhibitor fields. Through the nondimensionalization of the reaction-diffusion PDE (2.1), the $O(1)$ spatial scale is defined to be the natural diffusion length of the inhibitor $(v)$ field. In the limit of weak activator diffusion $(D \ll 1)$, the transitions of the activator field between bistable states are resolved within thin interfaces, or interior layers, whose $O(\sqrt{D})$ width is characteristic of diffusion-limited fronts. From the perspective of matched-asymptotics, the sharp activator fronts on the inner scale are contrasted by the more gradual relaxation of the inhibitor concentration that occurs on the outer scale.

Outer $O(1)$ scale. In one dimension $(x)$, the model equations (2.1) are rewritten explicitly as

$$
u(u-r)(u-1)=D u_{x x}-\rho(v-u)-u_{t},
$$




$$
v_{x x}-(v-u)=\epsilon v_{t},
$$

where the activator diffusion $(D \ll 1)$ and inhibition coupling $(\rho \ll 1)$ are assumed small. In anticipation of a dynamics consisting of slow front motion (implied by $D \ll 1$ ), both time derivatives may also be consistently assumed small. The right-hand sides of (3.14) are then seen to be perturbations with respect to this outer scale.

In terms of the outer variables, the underlying pattern is defined by regions where the activator is in either of its locally stable values. For the cubic nonlinearity (3.14a), these are

$$
u(x) \sim u^{0}(x)= \begin{cases}1 & \text { (black areas) } \\ 0 & \text { (white areas) }\end{cases}
$$

which defines a binary pattern on the line. The present approach is more general than the heuristic analysis of the previous section, capable of treating a pattern consisting of any number of fronts. The leading-order outer inhibition field $v^{0}(x)$ then satisfies the inhomogeneous elliptic equation

$$
v_{x x}^{0}-v^{0} \sim-u^{0}=- \begin{cases}1 & \text { (black areas) } \\ 0 & \text { (white areas) }\end{cases}
$$

where continuity of $v^{0}$ and $v_{x}^{0}$ is imposed at jump discontinuities in the binary pattern $u^{0}(x)$. As well, suitable boundary conditions at the domain edges (for example, periodic, or $v_{x}=0$ at infinity) must be included to insure a unique solution.

The time dependence of this leading-order outer solution is implicit in the functions $Q^{j}(t)$ that locate the boundary points between the black and white areas, the jump transitions of $u^{0}$. The velocity $Q_{t}^{j}$ of these interface points is obtained as a consequence of the asymptotic matching at inner scales to remove the discontinuities of the chemical fields.

Inner $O(\sqrt{D})$ scale. At each interface point $x=Q^{j}(t)$ the discontinuity of the outer activator solution $u^{0}(x)$ is resolved by an inner representation defined locally on a finer diffusion scale $\eta=\left(x-Q^{j}\right) / \sqrt{D}$. Since the activator solution is not discontinuous on this inner scale, the precise location of the interface $(\eta=0)$ is defined by the condition $u\left(x=Q^{j}\right)=1 / 2$. In terms of this rescaled and moving coordinate, the equations for the inner solutions $U(\eta, t)$ and $V(\eta, t)$ reflect a markedly different asymptotic ordering. Introducing the nonlinear operator $\mathcal{S}[U]$

$$
\mathcal{S}[U]=U_{\eta \eta}-U(U-1 / 2)(U-1),
$$

the inner equations become

$$
\begin{gathered}
\mathcal{S}[U]=-\frac{Q_{t}^{j}}{\sqrt{D}} U_{\eta}-6 \Delta F U(U-1)+\rho(V-U)+U_{t} \\
V_{\eta \eta}=D(V-U)+\epsilon D V_{t}
\end{gathered}
$$

The homogeneous solution of the left-hand sides of (3.18) correspond to a stationary front solution

$$
\begin{aligned}
U^{0}(\eta) & =\frac{1}{2}\left[1 \mp \tanh \left(\frac{\eta}{2 \sqrt{2}}\right)\right], \\
V^{0} & =v^{0}\left(x=Q^{j}\right),
\end{aligned}
$$

which, as $\eta \rightarrow \pm \infty$, match the behaviors of the outer solutions $u^{0}(x)$ and $v^{0}(x)$ at the interface $x \rightarrow\left(Q^{j}\right)^{ \pm}$. The sign $\mp$ is chosen by the orientation of the front, where a negative sign corresponds to a left-to-right transition from $u=1$ to $u=0$. Note also that, to leading-order, the inhibitor field is constant within this interior layer this is consistent with the continuity of the outer solution $v^{0}(x)$ of (3.16) which only requires an $O(D)$ transition solution to resolve the discontinuity in its second derivative.

The stationarity of this leading-order front (3.19) is broken by the effects of the right-side terms of (3.18) and suggests a perturbative derivation for the front speed $Q_{t}^{j}$ based on the asymptotic balance

$$
\frac{Q_{t}^{j}}{\sqrt{D}} \sim \Delta F \sim \rho \ll 1 .
$$

The determination of $Q_{t}^{j}$, which is essentially just a nonlinear eigenvalue for (3.18), then follows from the solvability condition for a (bounded) first correction to the inner activator solution

$$
U(\eta) \sim U^{0}(\eta)+\rho U^{1}(\eta)
$$

Substitution into (3.18a) gives an inhomogeneous ordinary differential equation (ODE) for the timeindependent first correction $U^{1}(\eta)$ involving the operator $\mathcal{S}^{\prime}\left[U^{1}\right]$ that is just the linearization of the nonlocal operator $\mathcal{S}[U](3.17)$

$$
\mathcal{S}^{\prime}\left[U^{1}\right]=U_{\eta \eta}^{1}-F^{\prime \prime}\left(U^{0} ; 1 / 2\right) U^{1},
$$

where the primes on $F$ denote differentiation on the first variable. The first-order perturbations are restricted to the terms involving the balance (3.20) and give

$$
\begin{aligned}
\rho \mathcal{S}^{\prime}\left[U^{1}\right]= & {\left[ \pm \frac{Q_{t}^{j}}{\sqrt{2 D}}+6 \Delta F\right] U^{0}\left(U^{0}-1\right) } \\
& +\rho\left[v^{0}\left(x=Q^{j}\right)-U^{0}\right] .
\end{aligned}
$$

Application of the identity $\sqrt{2} U_{\eta}^{0}=\mp U^{0}\left(U^{0}-1\right)$ has resulted in some simplification of terms in the above equation.

By the translation symmetry associated with the $U^{0}$ solution, the appropriate solvability condition for bounded solutions to (3.23) is the inner product integral

$$
\left\langle U_{\eta}^{0}, \bullet\right\rangle \equiv \int_{-\infty}^{+\infty} d \eta U_{\eta}^{0} \bullet
$$

Since the linearized operator $\mathcal{S}^{\prime}$ is orthogonal to $U^{0}$, the inner product of the equation (3.23) determines the unique front speed that ensures the existence of a bounded first correction $U^{1}(\eta)$,

$$
Q_{t}^{j} \sim \mp 6 \sqrt{2 D}\left\{\Delta F+\rho\left[v^{0}\left(x=Q^{j}\right)-\frac{1}{2}\right]\right\}
$$


where, again, the choice of sign $\mp$ is consistent with the choice made for the leading-order solution (3.19). Selfconsistently, the front velocity satisfies $Q_{t}^{j} \ll O \sqrt{D}$.

The important feature of the front speed result (3.25) is its dependence on the strength of the outer inhibition $v^{0}\left(x=Q^{j}\right)$. By the elliptic nature of the outer inhibitor equation (3.16), $v\left(x=Q^{j}\right)$ will, in general, be influenced by the location of other fronts, and hence embody the nonlocality. Thus, for a sequence of fronts, the speed relations (3.25) for $j=1$ to $N$ will be a set of $N$ coupled ODEs that describe the boundaries of the binary pattern for $u(x)$.

Motion of a single front. For this case, on the infinite line, we consider a pattern that is white $(u=0)$ to the right of an interface at $x=Q(t)$ and black $(u=1)$ to the left:

$$
u^{0}(x) \sim \begin{cases}1, & x \leq Q(t) \\ 0, & Q(t) \leq x\end{cases}
$$

Solving (3.16), the outer inhibitor field has the simple piecewise continuous solution

$$
v^{0}(x) \sim\left\{\begin{array}{l}
1-\frac{1}{2} e^{+(x-Q)}, \quad x \leq Q(t) \\
\frac{1}{2} e^{-(x-Q)}, \quad Q(t) \leq x
\end{array}\right.
$$

where $v_{x}=0$ boundary conditions are imposed at infinity. Since $v^{0}(x=Q)=\frac{1}{2}$ at the interface, by (3.25) there is seen to be no $O(\rho)$ correction to the front speed

$$
Q_{t} \sim-6 \sqrt{2 D} \Delta F,
$$

so that, when $\Delta F>0$, the front travels with constant negative (leftward) velocity. Motion towards the black region is the expected result when the white $(u=0)$ state is energetically preferred (2.2).

Higher-order corrections to the speed of a single front. The calculation of the front profile and speed may be continued to another order in $\rho$ in the case of a solitary front. The solution of (3.23) for the first correction $U^{1}$ is

$$
U^{1}(\eta)=-\frac{d}{d \eta}\left\{\eta \tanh \left(\frac{\eta}{2 \sqrt{2}}\right)\right\} .
$$

This describes the overshoot of the profile seen in Figs. 5 and 6 . The gradient of the front is increased in the neighborhood of $\eta=0$, and this would be expected to increase the rate of dissipation and hence retard the interface motion. This intuition is confirmed asymptotically when $\Delta F \sim \rho \gg \sqrt{D}$, as the next order of the solvability condition can be explicitly evaluated, giving the extended velocity condition

$$
Q_{t} \sim-6 \sqrt{2 D} \Delta F[1-6 \rho] .
$$

This linear correction to the front speed is clearly apparent in the plot of velocity versus $\rho$ in Fig. 7(a), obtained by direct numerical solution of the PDEs. Note also that beyond $\rho \simeq 0.02$, even this next-order asymptotic correction is insufficient for quantitative accuracy.

Interaction of two fronts. Reconsider the situation in an infinite domain $-\infty \leq x \leq+\infty$ in which two interfaces are symmetrically located at $x= \pm Q(t)$ between

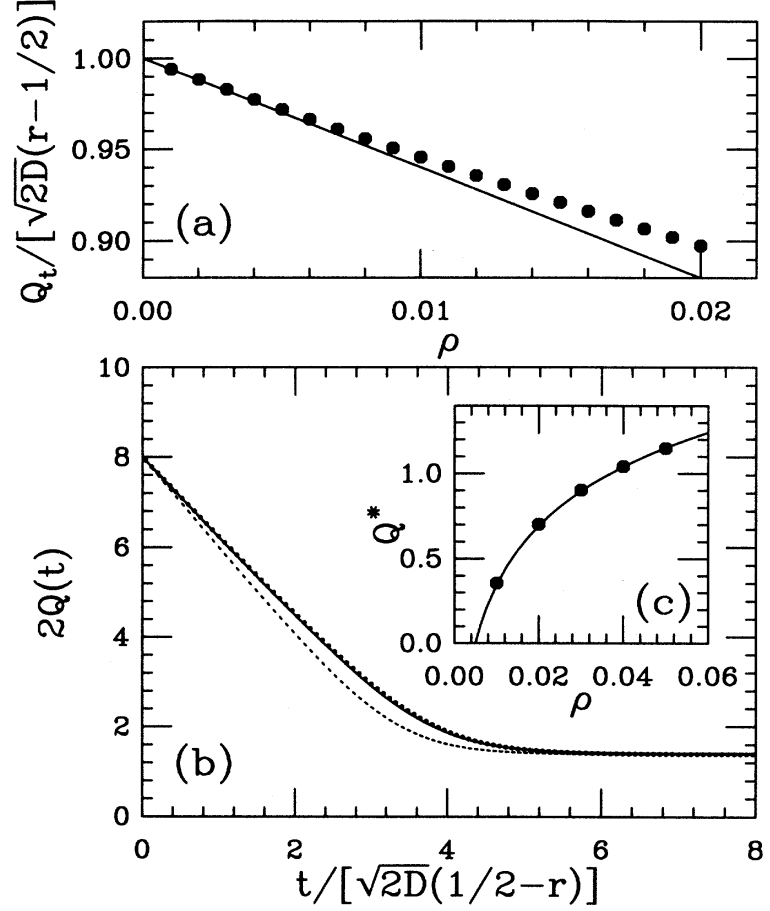

FIG. 7. Front dynamics. (a) Velocity of a single front as a function of the activator-inhibitor coupling, for diffusion constant $D=0.0001$, normalized by the velocity for $\rho=0$. Symbols indicate results of numerical simulations of the reaction-diffusion equations, while solid lines are predictions from the asymptotic analysis with the corrected friction coefficient given by Eq. (3.30). (b) Asymptotics for separation versus time for two approaching fronts in one dimension, compared with simulation results for $D=0.0001, r=0.515$, and $\rho=0.02$. The dashed line is prediction of asymptotics with bare friction coefficient, while the dotted line includes order $\rho$ correction to the front profile and compares well with the full numerical solution to the reaction-diffusion equations (solid line). Inset (c) shows the equilibrium separation of the fronts versus $\rho$ for both simulation (solid symbols) and asymptotics (solid line) from Eq. (3.11).

which $u=0$. These fronts are the edges of the localized state considered in the heuristic derivation which obtained the dynamics (3.10) - this result is confirmed below using the matched-asymptotic front speed formula (3.25).

The calculation proceeds as in the case of one front, but begins from the outer activator pattern

$$
u^{0}(x)=\left\{\begin{array}{l}
0, \quad-\infty<x \leq-Q(t) \\
1, \quad-Q(t) \leq x \leq+Q(t) \\
0, \quad+Q(t) \leq x<+\infty
\end{array}\right.
$$

that is symmetric about the origin. Solving (3.16), the outer inhibitor field can again be expressed in terms of exponential functions

$$
v^{0}(x)=\left\{\begin{array}{l}
\sinh Q e^{+x},-\infty<x \leq-Q(t) \\
1-e^{-Q} \cosh x,-Q(t) \leq x \leq+Q(t) \\
\sinh Q e^{-x},+Q(t) \leq x<+\infty
\end{array}\right.
$$


This determines the inhibitor contribution to the front speed

$$
v^{0}(x=Q)=\frac{1}{2}\left(1-e^{-2 Q}\right),
$$

where now, unlike in the case of a single front, $v^{0}(x=$ $\left.Q^{j}\right) \neq \frac{1}{2}$ at the interface. Substitution into the asymptotic formula for the front velocity (3.25) gives

$$
Q_{t} \sim-6 \sqrt{2 D}\left[\Delta F-\frac{\rho}{2} e^{-2 Q}\right]
$$

where the minus sign must be taken since $u\left(x=Q^{-}\right)=1$.

The result (3.34) not only recovers the heuristically derived stripe dynamics (3.10), but also determines the constant of proportionality associated with the line tension $\gamma$ (3.2) and the Rayleigh dissipation rate (3.9). Direct comparison of the two formulas (3.10) and (3.34) gives

$$
\gamma=\frac{1}{6} \sqrt{\frac{D}{2}}
$$

which is, as expected, $O(\sqrt{D})$. Moreover, since the line tension coefficient (3.2) represents the energetic contribution proportional to perimeter, the leading-order form of the front profile $U^{0}$ can also be used in the integration

$$
\gamma=\sqrt{D} \int_{-\infty}^{+\infty} d \eta\left\{\frac{1}{2}\left(U_{\eta}^{0}\right)^{2}+F\left(U^{0} ; \frac{1}{2}\right)\right\}
$$

Note that the additional contribution of $F\left(U ; \frac{1}{2}\right)$ is a boundary error associated with the pressure integral (3.3) and resolves the discrepancy involving the line tension energy that was alluded to below Eq. (3.6).

In addition to recovering the dynamics of stripe stabilization, the single front speed formula can be used to understand the behavior of two black stripes when $\Delta F>0$. If we were to start from a configuration in which the stripes were not at their equilibrium width, there would first be the ballistic collapse to form the black stripes of width near $2 Q^{*}$. On a slower time scale, the two stripes repel each other by their exponential tails of inhibition [on the $O(1)$ scale] - this is an illustration of the process of stripe repulsion [15].

\section{Numerical comparison}

Tests of the results for the dynamics and equilibrium separation of approaching fronts are shown in Fig. 7 for a situation like that in Fig. 5, but with the values $D=0.0001$ and $\rho \leq 0.02$, in the truly asymptotic regime. The front separation $2 Q$ is shown as a function of time and is compared with the predictions of Eq. (3.34), using both the bare prefactor $6 \sqrt{2 D}$ associated with the leading-order profile and the corrected prefactor $6 \sqrt{2 D}(1-6 \rho)$ of Eq. (3.30). We see that the latter leads to highly accurate results. The equilibrium separation between the fronts is independent of this prefactor and, as shown in Fig. 7(c), is in accurate agreement with the results of the numerical solution of the full partial differential equations.

\section{INTERFACE MOTION IN TWO DIMENSIONS}

\section{A. Heuristic derivation}

The heuristic derivation of the energetics and dynamics for contours in two dimensions proceeds along the lines used for one-dimensional patterns. This requires the additional assumption, beyond that of localized gradients, that the curvature of the boundary does not significantly affect the interface profile. We consider a single black domain $\mathcal{B}$ of $u=1$ surrounded by a sea of white, bounded by the contour $\mathbf{r}(s)$, where $s$ is the arclength. As in the calculation for one dimension, the energy functional $\Delta \mathcal{E}$ consists of three contributions.

The line tension contribution to the energy (2.18) must now involve an integral around the boundary of the black domain

$$
\oint_{\partial \mathcal{B}} d s \gamma=\gamma L
$$

where $L$ is the perimeter, and where the line tension $\gamma$ must be exactly that found earlier from the onedimensional asymptotics [Eq. (3.35)].

By analogy with the result in Eq. (3.3), the potential integral contributes an energy proportional to the domain area $A$,

$$
\int_{\mathcal{B}} d \mathbf{x}[F(1 ; r)-F(0, r)]=\Delta F A .
$$

The main difference from the one-dimensional problem is the treatment of the nonlocal term, which is proportional to the double integral $\iint \mathcal{G}$ over the domain. Substituting $\mathcal{G}=\nabla^{2} \mathcal{G}+\delta\left(\mathbf{x}-\mathbf{x}^{\prime}\right)$ we may recast the bulk contributions as boundary terms by twice using the Green's identity $\int_{A} \boldsymbol{\nabla} \psi=\int_{\partial A} \hat{\mathbf{n}} \psi$ in a manner analogous to that in Eqs. (3.4) and (3.5) as follows:

$$
\begin{aligned}
\int_{\mathcal{B}} d \mathbf{x} \int_{\mathcal{B}} d \mathbf{x}^{\prime} \mathcal{G}-A & =-\int_{\mathcal{B}} d \mathbf{x} \nabla_{\mathbf{x}} \cdot \int_{\mathcal{B}} d \mathbf{x}^{\prime} \boldsymbol{\nabla}_{\mathbf{x}^{\prime}} \mathcal{G} \\
& =-\int_{\mathcal{B}} d \mathbf{x} \boldsymbol{\nabla}_{\mathbf{x}} \cdot \oint_{\partial \mathcal{B}} d s^{\prime} \hat{\mathbf{n}}\left(s^{\prime}\right) \mathcal{G} \\
& =-\oint_{\partial \mathcal{B}} d s \oint_{\partial \mathcal{B}} d s^{\prime} \hat{\mathbf{n}}(s) \cdot \hat{\mathbf{n}}\left(s^{\prime}\right) \mathcal{G}
\end{aligned}
$$

Note that $\hat{\mathbf{n}}(s)$ is the unit normal vector pointing out of the black domain. Finally, collecting all of the terms and noting that $\hat{\mathbf{n}}(s) \cdot \hat{\mathbf{n}}\left(s^{\prime}\right)=\hat{\mathbf{t}}(s) \cdot \hat{\mathbf{t}}\left(s^{\prime}\right)$, where $\hat{\mathbf{t}}$ is the unit tangent vector, we obtain

$\Delta \mathcal{E}[\mathbf{r}]=\gamma L+\Delta F A-\frac{1}{2} \rho \oint d s \oint d s^{\prime} \hat{\mathbf{t}}(s) \cdot \hat{\mathbf{t}}\left(s^{\prime}\right) \mathcal{G}\left(\mathbf{r}-\mathbf{r}^{\prime}\right)$

Unlike in one dimension, the nonlocality cannot be reduced to a pointwise evaluation of the Green's function. 
The form of the nonlocal coupling between tangent vectors is reminiscent of the self-induction of currentcarrying wires, the direction of the current being specified by $\hat{\mathbf{t}}$. Indeed, the sign of the interaction is such that antiparallel tangent vectors repel (like antiparallel current-carrying wires), while parallel ones attract. An alternative view of this connection with electromagnetic systems is to consider the inhibitor relation (2.14) as a Poisson equation relating a potential $(v)$ to a charge density $(u)$, in which case the energy function $\mathcal{F}=\int u v$ in Eq. (2.11) is the associated electrostatic energy. Through the correspondence between magnetization and current loops, and the similarity of electric and magnetic dipolar phenomena, such a nonlocal interaction appears in the description of pattern formation in a variety of other systems, as detailed in Sec. XIII. In such dipolar systems an important role is played by molecular or other cutoffs in the self-induction integrals. The present formulation neither has such cutoffs nor needs one for a well-defined energy, as the singularity in the Green's function $\mathcal{G}$ in (4.4) as $s-s^{\prime} \rightarrow 0$ is integrable, being only logarithmic in $\left|s-s^{\prime}\right|$.

With the energy now formulated as a functional of the boundary contour $\mathbf{r}$, it only remains to calculate the Rayleigh dissipation functional. This is just the integral around the boundary of the one-dimensional result

$$
\mathcal{R}=\frac{\gamma}{2 D} \oint_{\partial \mathcal{B}} d s\left(\hat{\mathbf{n}} \cdot \mathbf{r}_{t}\right)^{2}
$$

To obtain the functional derivative of the energy (4.4), we combine the local contributions

$$
\begin{aligned}
& \frac{1}{\sqrt{g}} \frac{\delta \mathcal{R}}{\delta \mathbf{r}_{t}}=\frac{\gamma}{D}\left(\hat{\mathbf{n}} \cdot \mathbf{r}_{t}\right) \hat{\mathbf{n}} \\
& \frac{1}{\sqrt{g}} \frac{\delta L}{\delta \mathbf{r}}=\kappa \hat{\mathbf{n}} \\
& \frac{1}{\sqrt{g}} \frac{\delta A}{\delta \mathbf{r}}=\hat{\mathbf{n}}
\end{aligned}
$$

where $g$ is the metric, with the result for a self-induction integral $[38,39]$

$$
\begin{aligned}
& \frac{\delta}{\delta \mathbf{r}} \frac{1}{2} \oint d s \oint d s^{\prime} \hat{\mathbf{t}} \cdot \hat{\mathbf{t}}^{\prime} \mathcal{G}(R) \\
& \quad=\left\{\oint d s^{\prime} \hat{\mathbf{R}}\left(s, s^{\prime}\right) \times \hat{\mathbf{t}}^{\prime} \mathcal{G}^{\prime}(R)\right\} \hat{\mathbf{n}}(s),
\end{aligned}
$$

where $\hat{\mathbf{R}}\left(s, s^{\prime}\right)=\left[\mathbf{r}(s)-\mathbf{r}\left(s^{\prime}\right)\right] /\left|\mathbf{r}(s)-\mathbf{r}\left(s^{\prime}\right)\right|$, the cross product is a scalar in two dimensions $\left(\mathbf{a} \times \mathbf{b}=\epsilon_{i j} a_{i} b_{j}\right)$, and the prime on $\mathcal{G}$ indicates differentiation: $\mathcal{G}^{\prime} \equiv$ $d \mathcal{G} / d R=-K_{1}(R)$. The variational principle (3.8) determines the contour evolution, expressed as the normal velocity

$\hat{\mathbf{n}} \cdot \mathbf{r}_{t}=-\frac{D}{\gamma}\left\{\gamma \kappa(s)+\Delta F-\rho \oint d s^{\prime} \hat{\mathbf{R}}\left(s, s^{\prime}\right) \times \hat{\mathbf{t}}^{\prime} \mathcal{G}^{\prime}(R)\right\}$.

Note that the nonlocal contribution in (4.8) is not a singular integral, since the integrand for $s^{\prime} \rightarrow s$ has the

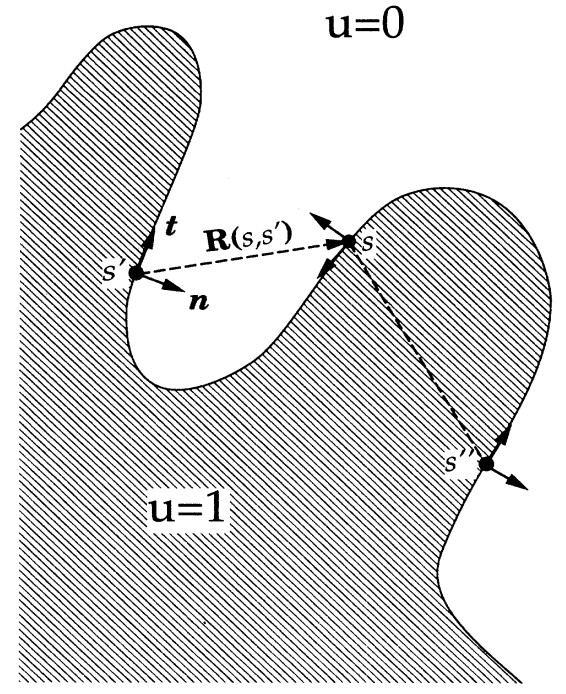

FIG. 8. Illustration of the Biot-Savart interactions of nearby chemical fronts, as described by the nonlocal contribution to the force law in Eq. (4.8.).

finite limiting value

$$
\lim _{s^{\prime} \rightarrow s}\left\{\hat{\mathbf{R}}\left(s, s^{\prime}\right) \times \hat{\mathbf{t}}\left(s^{\prime}\right) K_{1}\left[\left|\mathbf{r}(s)-\mathbf{r}\left(s^{\prime}\right)\right|\right]\right\}=-\frac{1}{2} \kappa(s) .
$$

It is readily verified from Eq. (4.8) and Fig. 8 that the vector product in the normal velocity embodies the repulsion between nearby antiparallel sections of the contour. As noted in earlier work [10], this repulsion between adjacent fronts requires only that $\mathcal{G}>0$ and $\mathcal{G}^{\prime}<0$ (for $\rho>0$ ), and not on the details of $\mathcal{G}$.

\section{B. Asymptotic derivation of a curvature dynamics}

Now we rederive the contour dynamics (4.8) by adapting the asymptotic argument presented earlier for one spatial dimension. The only major modification involves the reorientation of the the inner coordinate $(\eta)$ of the activator front to be in the direction normal to the interface.

Outer $O(1)$ scale. In two dimensions, $\mathbf{x}=(x, y)$, the leading-order outer representation satisfies the left-hand side of the equations

$$
\begin{gathered}
u(u-r)(u-1)=D \nabla^{2} u-\rho(v-u)-u_{t} \\
\nabla^{2} v-(v-u)=\epsilon v_{t} .
\end{gathered}
$$

The parameter scalings are chosen to obtain the analogous front dynamics, but with the added dimensionality, the activator diffusion scale $(\sqrt{D})$ is incorporated into the significant limit

$$
\frac{Q_{t}}{\sqrt{D}} \sim \Delta F \sim \rho \sim \sqrt{D} \ll 1 .
$$

As before, the underlying pattern is defined by patches 
where the activator is in either of its bistable states

$$
u(\mathbf{x}) \sim u^{0}(\mathbf{x})= \begin{cases}1 & \text { (black areas) } \\ 0 & \text { (white areas) }\end{cases}
$$

Unlike in one dimension, where the transitions between black and white are points, in two dimensions the interface separating a black patch from a white background is defined by a (slowly moving) contour in the $(x, y)$ plane,

$$
\mathbf{r}(\alpha ; t)=(X(\alpha ; t), Y(\alpha ; t)),
$$

where $\alpha$ is defined as a counterclockwise (but not necessarily arclength) parameterization around the black patch. There may, of course, be more than one disjoint black patch, in which case multiple contours $\mathbf{r}^{j}$ must be evolved. For simplicity of presentation, however, these indices are omitted. The leading-order inhibition field $v^{0}(\mathbf{x})$ then satisfies the inhomogeneous elliptic equation with a two-dimensional Laplacian

$$
\nabla^{2} v^{0}-v^{0} \sim-u^{0}=-\left\{\begin{array}{ll}
1 & \text { (black areas) } \\
0 & \text { (white areas) }
\end{array} .\right.
$$

Along the interface $\mathbf{r}$, continuity is imposed on $v^{0}$ and on $(\hat{\mathbf{n}} \cdot \nabla) v^{0}$, the normal derivative. Again, with suitable boundary conditions at the domain edges, a unique solution is guaranteed. For consistency with the separation of scales assumed in the asymptotics, it is essential that the interface geometry contain structure only on the inhibitor diffusion length - this demands that both the (global) interfacial separation distances and the (local) radius of curvature be $O(1)$.

Local orthogonal coordinates. The discontinuity of the activator on the outer scale is naturally resolved on an inner scale, which is a stretched coordinate normal to the interface. At a point along the contour $\mathbf{r}(\alpha ; t)$, the local (unnormalized) tangent (t) and normal (n) directions are given by

$$
\begin{aligned}
& \mathbf{t}=\mathbf{r}_{\alpha} \\
& \mathbf{n}=\mathbf{J r}_{\boldsymbol{\alpha}}=\left[\begin{array}{cc}
0 & +1 \\
-1 & 0
\end{array}\right] \mathbf{r}_{\alpha}
\end{aligned}
$$

where the matrix $\mathrm{J}$ corresponds to a $90^{\circ}$ clockwise rotation. With the counterclockwise orientation of the parameterization $\alpha$, the normal $\mathbf{n}$ points into regions of white. Using these as basis vectors for a local coordinate system, introduce a new coordinate $\bar{\eta}$ that extends orthogonally from the contour $\mathbf{r}$ in the direction of the normal (n). This suggests the change of variable (suppressing the time dependence)

$$
\mathbf{x}(\bar{\eta}, \alpha)=\mathbf{r}(\alpha)+\bar{\eta} \mathbf{J} \mathbf{r}_{\alpha}(\alpha)
$$

so that $\bar{\eta}=0$ coincides with the contour $\mathbf{r}$. In the thin interface limit, the inner solution requires the validity of local coordinates only for small $\bar{\eta}=O(\sqrt{D})$. It proves calculationally more convenient to use instead an infinite (Lie) series as the change of variable

$$
\begin{aligned}
\mathbf{x}(\bar{\eta}, \alpha) & =\mathbf{r}+\bar{\eta} \mathbf{J} \mathbf{r}_{\alpha}+\frac{1}{2} \bar{\eta}^{2} \mathbf{J}^{2} \mathbf{r}_{\alpha \alpha}+\cdots \\
& \equiv \exp \left\{\bar{\eta} \mathbf{J} \frac{\partial}{\partial \alpha}\right\} \mathbf{r}
\end{aligned}
$$

for which (4.17) is the linearization. Straightforward differentiation demonstrates that the complete series (4.19) has the property that it is orthogonal even for $\bar{\eta} \neq 0$ off the generating contour $\mathbf{r}$

$$
\mathbf{x}_{\tilde{\eta}}=\mathbf{J} \mathbf{x}_{\alpha}
$$

These are just the Cauchy-Riemann conditions, thereby showing that the change of variables between $(x, y)$ and $(\bar{\eta}, \alpha)$ is locally conformal. Although for small $\bar{\eta}$ only a few terms in this series are necessary in the present analysis, it establishes to all orders that the Laplacian nature of the diffusions is preserved up to a Jacobian metric,

$$
\nabla_{x, y}^{2}=\frac{1}{\left|\mathbf{x}_{\alpha}(\bar{\eta}, \alpha ; t)\right|^{2}} \nabla_{\bar{\eta}, \alpha}^{2}
$$

Inner $O(\sqrt{D})$ scale. The resolution of the activator front, as well as the front speed determination, now follows analogously to the case of one dimension. The stretched inner coordinate $\eta$,

$$
\eta=\frac{1}{\sqrt{D}} \frac{\bar{\eta}}{\left|\mathbf{r}_{\alpha}\right|}
$$

resolves the activator front across the discontinuity, while the $\alpha$-coordinate labels position along the contour. Using the expansion (4.19), we find that the metric factor in $(4.21)$ is

$$
\left|\mathbf{x}_{\alpha}(\bar{\eta}, \alpha ; t)\right|^{2} \sim\left|\mathbf{r}_{\alpha}\right|^{2}[1+2 \sqrt{D} \eta \kappa+\cdots],
$$

where we have used the general expression for the curvature $\kappa$,

$$
\kappa=\hat{\mathbf{t}} \cdot \hat{\mathbf{n}}_{s}=\frac{\mathbf{r}_{\alpha} \cdot \mathbf{J} \mathbf{r}_{\alpha \alpha}}{\left|\mathbf{r}_{\alpha}\right|^{3}}
$$

defined such that it is positive for a convex black domain. Note that although the $\left|\mathbf{r}_{\alpha}\right|$ normalization weakly breaks the orthogonality property, up to the first correction in curvature the Laplacian has the simple form

$$
\nabla_{x, y}^{2} \sim \frac{1}{D}(1-2 \sqrt{D} \eta \kappa) \frac{\partial^{2}}{\partial \eta^{2}}+O(1)
$$

At each point along the interface $\mathbf{r}(\alpha)$, the normal component of the velocity is sufficient to describe the interface motion. This freedom essentially follows from an arbitrariness in the contour parameterization $\alpha$, which permits arbitrary choices of tangential velocity. Here we may choose the parameterization that yields, at fixed $\alpha$, an interface moving normal to itself, with $Q_{t}(\alpha ; t)$ the leading-order normal velocity,

$$
Q_{t}(\alpha ; t)=\hat{\mathbf{n}} \cdot \mathbf{r}_{t}
$$


At the level of the first correction, the inner equations for the front profile are identical to the one-dimensional case (3.18), but with the addition of a curvature term from the Laplacian expansion (4.25),

$$
\begin{gathered}
\mathcal{S}[U] \sim-\frac{Q_{t}}{\sqrt{D}} U_{\eta}+2 \sqrt{D} \kappa \eta U_{\eta \eta}-6 \Delta F U(U-1) \\
+\rho(V-U)+O(D) \\
V_{\eta \eta}=O(D)
\end{gathered}
$$

where $\mathcal{S}$ is as defined above (3.17). The leading-order solution $U^{0}(\eta)$ is identical to the hyperbolic tangent solution (3.19). Application of the same solvability inner product (3.24) yields the front speed relation

$Q_{t} \sim-6 \sqrt{2 D}\left\{\frac{1}{6} \sqrt{\frac{D}{2}} \kappa+\Delta F+\rho\left[v^{0}(x=Q)-\frac{1}{2}\right]\right\}$

which clearly recovers the curvature and potential effects of the energetically determined front velocity (4.8). In order for the two expressions to be in complete agreement, the nonlocal contribution of the contour integral in (4.8) must then be equivalent to the contribution from the inhibition term $v^{0}(x=Q)$ in (4.28).

The nonlocal character of the inhibitor field on the pattern is expressed mathematically by the Green's function solution of the outer inhibitor equation (4.14)

$$
v(\mathbf{x})=-\frac{1}{2 \pi} \int_{\mathcal{B}} d \mathbf{x}^{\prime} K_{0}\left(\left|\mathbf{x}-\mathbf{x}^{\prime}\right|\right),
$$

where the integration is done only over black areas. Using the PDE for $K_{0}$ and Green's theorem, the above solution can be rewritten in terms of a line integral over the interfacial boundary $\mathbf{r}$. This results in the arclength integral

$$
v(\mathbf{r})=\frac{1}{2}+\frac{1}{2 \pi} \oint d s^{\prime} \hat{\mathbf{n}} \cdot \frac{\mathbf{r}-\mathbf{r}^{\prime}}{\left|\mathbf{r}-\mathbf{r}^{\prime}\right|} K_{1}\left(\left|\mathbf{r}-\mathbf{r}^{\prime}\right|\right),
$$

where $K_{1}$ is introduced via the Bessel identity $K_{1}=$ $-K_{0}^{\prime}$. It should be noted that a Plemelj-type argument [40] is required to evaluate this integral, since $v^{0}$ must be evaluated for a point on the boundary $\mathbf{r}$. Here however, the usual principal value integral is unnecessary because the singularity in the integrand is removable [see Eq. (4.9)].

Substitution of the contour integral (4.30) into the asymptotic front speed (4.28) confirms the energetic result (4.4). In terms of $v^{0}(x=Q)$, the global energy of a black pattern may be written as

$$
\mathcal{E}=\gamma L+\frac{\rho}{2} \int_{\mathcal{B}} d \mathbf{x}^{\prime}\left[v^{0}(\mathbf{x})-1+\frac{2 \Delta F}{\rho}\right],
$$

a result that is not immediately obvious from the asymptotic derivation. With the area $A$ expressed as an integral, we see immediately from $v^{0}-1 \leq 0$ that the inte- grand loses positive definiteness for $\rho>2 \Delta F$, which is precisely the criterion for the stability of stripes [cf. Eq. (3.11)].

As formulated here, the front velocity formulas (4.28) and (4.4) both involve the evaluation of the outer inhibitor field, either by boundary integration (4.3), or direct solution of the bulk PDE (4.14). The boundary approach offers a compact, intrinsic description of complex contours; however, the bulk PDE approach can be calculationally advantageous in simple geometries where natural eigenfunctions can be constructed. An example of the latter is the analysis of a disk-shaped domain.

Localized disk solution. For a black circular spot of radius $R$ in an infinite white domain, the outer inhibitor $v^{0}$ has a radially symmetric solution of (4.14) in terms of modified Bessel functions

$$
v^{0}(r)=\left\{\begin{array}{l}
1-R K_{1}(R) I_{0}(r), \quad r \leq R \\
R I_{1}(R) K_{0}(r), \quad R \leq r
\end{array}\right.
$$

where continuity of $v^{0}$ and $v_{r}^{0}$ is satisfied at $r=R$. Direct substitution of $v^{0}(R)$ into (4.28) gives a nonlinear ODE for the purely radial evolution of the contour

$$
\begin{aligned}
R_{t} \sim & -6 \sqrt{2 D}\left\{\frac{1}{6}\left(\frac{D}{2}\right)^{1 / 2} \frac{1}{R}+\Delta F\right. \\
& \left.+\rho\left[R I_{1}(R) K_{0}(R)-\frac{1}{2}\right]\right\} .
\end{aligned}
$$

The dynamics (4.33) shows that the condition for the existence and radial stability of equilibrium disk solutions depends only on two $O(1)$ parameters: the potential difference and the inhibitor coupling, both scaled on the activator diffusion length. Equilibrium radii $R^{*}$ then satisfy the transcendental condition

$$
\frac{\sqrt{D}}{6 \sqrt{2} R^{*}}+\Delta F+\rho\left[R^{*} I_{1}\left(R^{*}\right) K_{0}\left(R^{*}\right)-\frac{1}{2}\right]=0
$$

which, upon numerical root tracking, identifies four distinct equilibrium scenarios. These are illustrated in Fig. 9: (a) two equilibria, only larger radius stable; (b) one equilibrium, unstable; (c) no equilibria, radial contraction only; (d) three equilibria, intermediate disk stable. Apart from the anomalous region (d), note that black localized disk patterns can only be stabilized when $\Delta F>0$ and white is energetically preferred. This behavior is the analog of the one-dimensional stripe stabilization and illustrates the process of domain localization. Although stable disks can be sustained in the small region (d) for $\Delta F<0$, since this region is so small in parameter space and the attracting basin for the stable radius relatively narrow, we believe these states to be unimportant, at least in the context of this particular formulation of the FitzHugh-Nagumo system.

It is also important to note that in the absence of inhibitor coupling, no stable disks are possible. The case in which $\Delta F=0$ (and $\rho=0$ ) corresponds to the wellknown shrinkage by curvature that leads to a singular collapse in finite time 


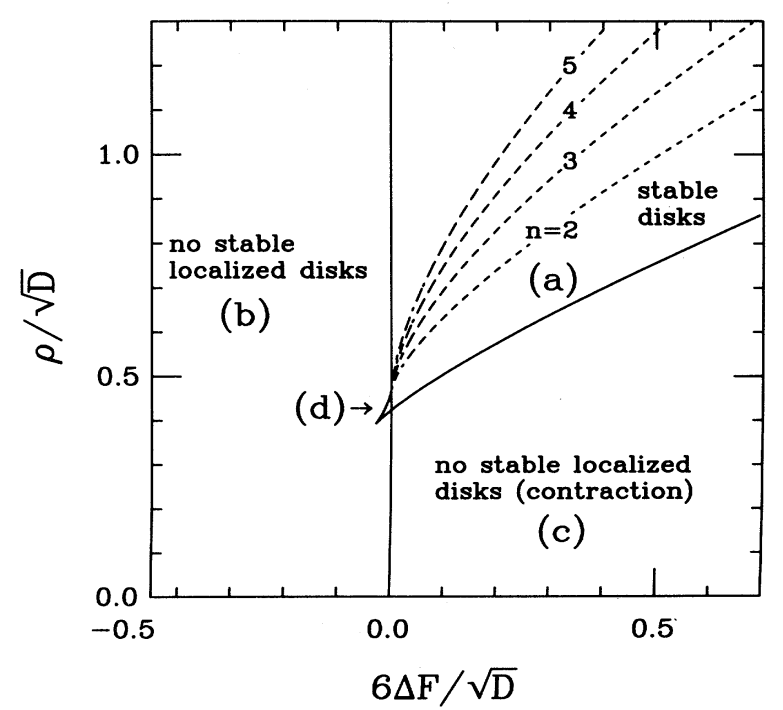

FIG. 9. Regions of existence and marginal stability curves of circular localized states in a rescaled parameter space, as deduced from the equilibrium condition of Eq. (4.34) and the linear stability result of Eq. (5.23).

$$
R(t) \sim \sqrt{2 D\left(t_{0}-t\right)} .
$$

Note that the PDE evolutions shown in Figs. 2 and 3 both have parameter values that lie within region (a). The dynamical evolution in (4.34) is restricted to radially symmetric geometries; the possibility of occurrence of azimuthal instabilities, which ultimately generate the labyrinthine patterns, is addressed in the following section.

Just as we obtained the energy of a domain in one dimension and deduced the existence of an energy barrier to its creation, we may compute the energy of a circular domain of radius $R$ on the basis of Eq. (4.4). The computation of the nonlocal contribution may be done two ways, via the direct calculation of the self-induction integral and by finding the inhibitor field $v(r)$ associated with a circular activator pulse. Collecting all of the contributions, we obtain the energy of a circle of radius $R$,

$$
\Delta \mathcal{E}(R)=2 \pi R \gamma+\pi R^{2} \Delta F-\pi \rho R^{2} K_{1}(R) I_{1}(R) .
$$

Using the simplest estimate of $\gamma$ from Eq. (3.35), Fig. 10 shows for several different values of $\rho$ the function $\Delta \mathcal{E}(R)$ in Eq. (4.36), illustrating the presence of a local minimum at a finite value of $R$ for sufficiently large $\rho$.

\section{INSTABILITIES OF CHEMICAL FRONTS}

\section{A. Mechanism of the transverse front instability}

The last of the three important aspects of the reactiondiffusion dynamics outlined in the Introduction concerns the instabilities of chemical fronts. At the level of linear stability analysis, we will see that the mechanism of this instability is rather similar to that of the Mullins-Sekerka

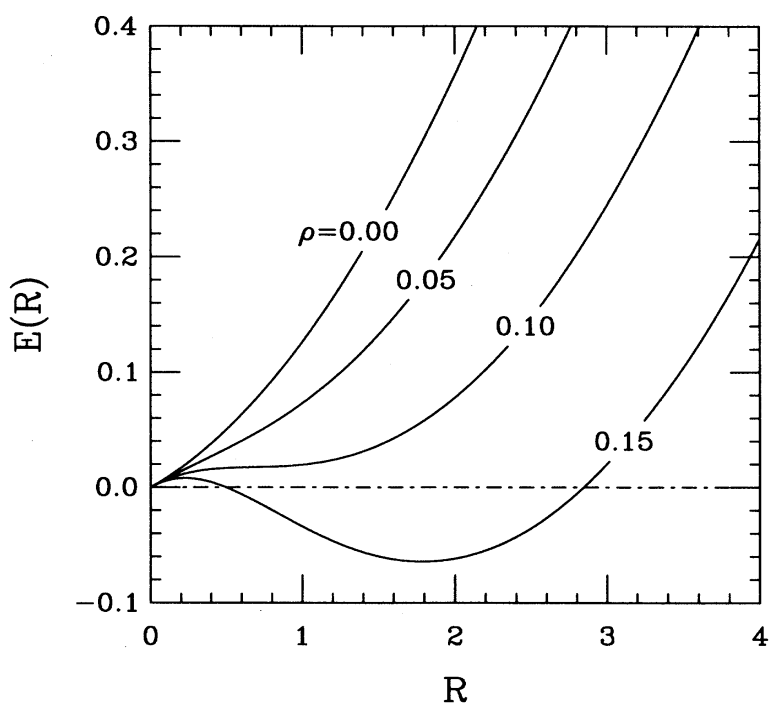

FIG. 10. Energy of circular domains as a function of radius, from Eq. (4.36), for various values of the activator-inhibitor coupling $\rho$, with $D=0.01$ and $r=0.6$.

instability of a liquid-solid interface [41]. In Fig. 11 we show schematically the level curves of activator and inhibitor concentration near a modulated interface of the inhibitor. The excess of inhibitor in the concave regions of the front has the tendency to push those concavities further back. Conversely, the portions of the front that are convex outwards are locally depleted in inhibitor relative to the flat interface, and will expand further outward. Both of these effects increase the length of the interface and its curvature, and will thus be resisted by

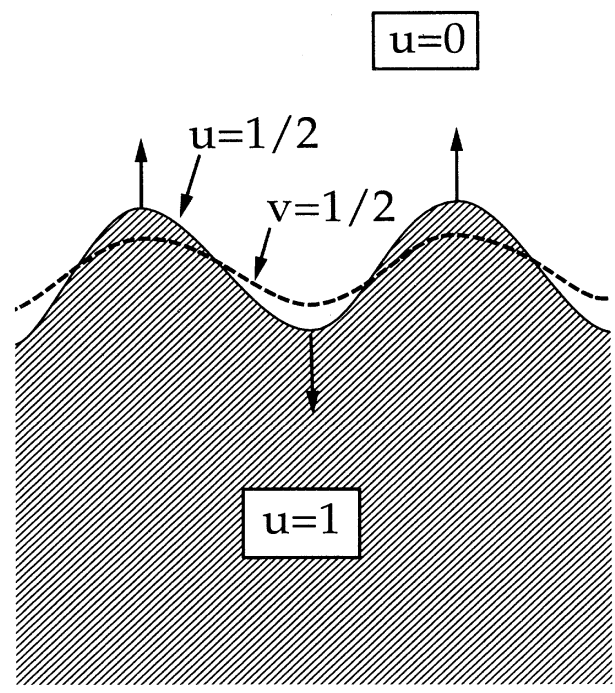

FIG. 11. Mechanism of instability of a straight chemical front. The accumulation of inhibitor in the neighborhood of segments of the interface that are concave inwards leads to the deepening of the those deformations, and vice-versa for regions that are convex outwards. 
line tension. The two effects will balance at a characteristic length scale to produce an instability.

The instability may be understood in its simplest form at the level of the energetics of circular domains; consider again the expression in Eq. (4.36) and examine the local limit $1 / R \ll 1$. In this limit, we use the asymptotics $K_{1}(z) I_{1}(z) \simeq(1 / 2 z)\left(1-3 / 8 z^{2}+\cdots\right)$ to simplify the energy

$$
\Delta \mathcal{E}=2 \pi R\left(\gamma-\frac{1}{4} \rho\right)+\pi R^{2} \Pi+\frac{3 \pi \rho}{16 R}+\cdots .
$$

We see two consequences of the inhibitor coupling. First there is an effective line tension $\gamma-\rho / 4$ which may be driven negative for sufficiently large activator-inhibitor coupling. Clearly, a negative line tension will lead to proliferation of the interface. A negative line tension is a common feature of fingering instabilities arising from the competition between Young-Laplace and Biot-Savart interactions $[20,39,42,43]$. Second, at higher order in $1 / R$ there appears a stabilizing contribution which below we show is like that for the bending energy of an elastic rod. If the effective tension is negative, then this last contribution will stabilize the interface against small-scale disturbances and produce a finite-wavelength instability.

\section{B. Approximate local contour dynamics}

The heuristic notion of an effective line tension may be made more systematic by recasting the nonlocal contour dynamics in an approximate local (but still intrinsic) form through an expansion in powers of the presumed small curvature $\kappa$ and its arclength derivatives. The method of this expansion is similar in spirit to that used recently [44] to study screened electrostatic potentials near surfaces of arbitrary geometry. Indeed, the inhibitor field obeys the same modified Helmholtz operator as appears in the Debye-Hückel theory of colloidal interactions [45], which leads to the screened Coulomb interaction between elementary charges.

The curvature expansion may be constructed directly from the equation of motion, or first at the level of the energy functional and then carried through the variational principle. Adopting the latter method, we first expand the scalar product of tangent vectors in the self-induction integral (4.4) as a power series in $\Delta=s^{\prime}-s$,

$$
\hat{\mathbf{t}}(s) \cdot \hat{\mathbf{t}}\left(s^{\prime}\right) \simeq 1-\frac{1}{2} \Delta^{2} \kappa^{2}+\cdots,
$$

and the distance function

$$
\left|\mathbf{r}\left(s^{\prime}\right)-\mathbf{r}(s)\right| \simeq|\Delta|-\frac{1}{24} \Delta^{2}|\Delta| \kappa^{2}+\cdots .
$$

Substituting into the self-induction integrals and extending the limits of integration over $s^{\prime}$ to $\pm \infty$ (with exponential accuracy), we obtain

$$
\begin{aligned}
& \oint d s \oint d s^{\prime} \hat{\mathbf{t}} \cdot \hat{\mathbf{t}}^{\prime} K_{0}\left(\left|\mathbf{r}-\mathbf{r}^{\prime}\right|\right) \\
& \simeq \pi \oint d s\left(1-\frac{3}{8} \kappa^{2}(s)+O\left(\kappa^{4}, \kappa_{s s}\right)\right)
\end{aligned}
$$

and the approximate local energy functional

$$
\Delta \mathcal{E}[\mathbf{r}] \simeq \Pi A+\bar{\gamma} L+\frac{3}{32} \rho \oint d s \kappa^{2},
$$

where the effective line tension is as in Eq. (5.1): $\bar{\gamma}=$ $\gamma-(1 / 4) \rho$. The term proportional to $\kappa^{2}$ in Eq. (5.5) is the bending energy of an elastic line [46] and its coefficient is positive if the activator-inhibitor coupling constant $\rho$ is positive. Under that condition, it prevents the interface from bending on arbitrarily fine scales.

Using the approximate local energy functional (5.5), the normal velocity of the interface is

$$
\frac{\gamma}{D} \hat{\mathbf{n}} \cdot \mathbf{r}_{t} \simeq-\Delta F-\bar{\gamma} \kappa+\frac{3}{16} \rho\left(\kappa_{s s}+\frac{1}{2} \kappa^{3}\right) .
$$

Apart from the pressure term, this is the planar version of the "curve-straightening equation" [47] that is equivalent to the Rouse dynamics of the wormlike model of elastic polymers [48]. It has the form of a Landau expansion in powers of the curvature and its derivatives, and in that sense is similar to so-called "geometric" models of crystal growth [49]. Unlike those models, however, the coefficients of the various terms in Eq. (5.6) are not all independent. Thus, for instance, the terms $\kappa_{s s}$ and $\kappa^{3}$ must have coefficients with ratio 2 by virtue of the variational principle applied to the energy functional (5.5).

\section{Linear stability for fronts and stripes}

The most fundamental instability of chemical fronts in the present model is that of an infinite straight interface bounding the states $u=0$ and $u=1$. To compute the spectrum of growth rates of perturbations to this front, we parametrize the interface as

$$
\mathbf{r}(s)=x \hat{\mathbf{e}}_{x}+\zeta(x, t) \hat{\mathbf{e}}_{y} .
$$

If we let $\zeta(x, t)=\zeta_{k}(t) \cos (k x)$, the linearization of the Biot-Savart integral is

$$
\begin{aligned}
& \oint d s^{\prime} \hat{\mathbf{R}} \times \hat{\mathbf{t}}\left(s^{\prime}\right) K_{1}(R) \\
& \simeq 2 \zeta_{k} \cos (k x) \\
& \quad \times \int_{0}^{\infty} d y\left\{\frac{k y \sin (k y) \cos (k y)-1}{y}\right\} K_{1}(y) .
\end{aligned}
$$

Use of the identity $(1 / y) K_{1}(y)=-K_{0}(y)-K_{1}^{\prime}(y)$ and an integration by parts transforms (5.8) into a standard integral. Combining these results with the linearized curvature, the growth rate $\sigma(k)=\left(\partial_{t} \zeta_{k}\right) / \zeta_{k}$ for the single front instability is

$$
\sigma_{\mathbf{f}}(k)=6 \sqrt{2 D}\left(-\gamma k^{2}+\frac{\rho}{2}\left[1-\frac{1}{f(k)}\right]\right),
$$

where

$$
f(k)=\sqrt{1+k^{2}}
$$


For wavelengths that are small relative to the $O(1)$ inhibitor screening length, we obtain

$$
\sigma_{\mathrm{f}}(k) \simeq 6 \sqrt{2 D}\left(-\left[\gamma-\frac{1}{4} \rho\right] k^{2}-\frac{3}{16} \rho k^{4}+\cdots\right),
$$

showing, as discussed in the preceding section [see in particular Eq. (5.6)], that the instability arises from a negative effective line tension, stabilized by an elastic-type term in $k^{4}$. This result also shows that at the onset of the instability the critical wavelength is infinite. As in the analysis of steady states for disk solutions, Eq. (4.34), we shall find it convenient to work in a rescaled parameter space with coordinates $\tilde{\rho}$ and $\tilde{r}$ as in (3.13). Setting $\sigma(k)=0$ and $d \sigma(k) / d k^{2}=0$ we obtain the critical coupling constant for the instability,

$$
\tilde{\rho}_{f}=\frac{4 \gamma}{\sqrt{D}}=\frac{\sqrt{2}}{3} .
$$

Note that this is independent of the parameter $r$, and hence also of the energy difference $\Delta F$, and so is equally valid as a stability criterion for a uniformly moving straight front. The critical value of $\rho$ could also be obtained directly from the vanishing of the effective line tension in (5.11). For $\rho>\rho_{f}$, the most unstable wave vector is

$$
k^{*}=\left[\left(\frac{\rho}{\rho_{f}}\right)^{2 / 3}-1\right]^{1 / 2} .
$$

Figure 12 shows in the rescaled parameter space the lo-

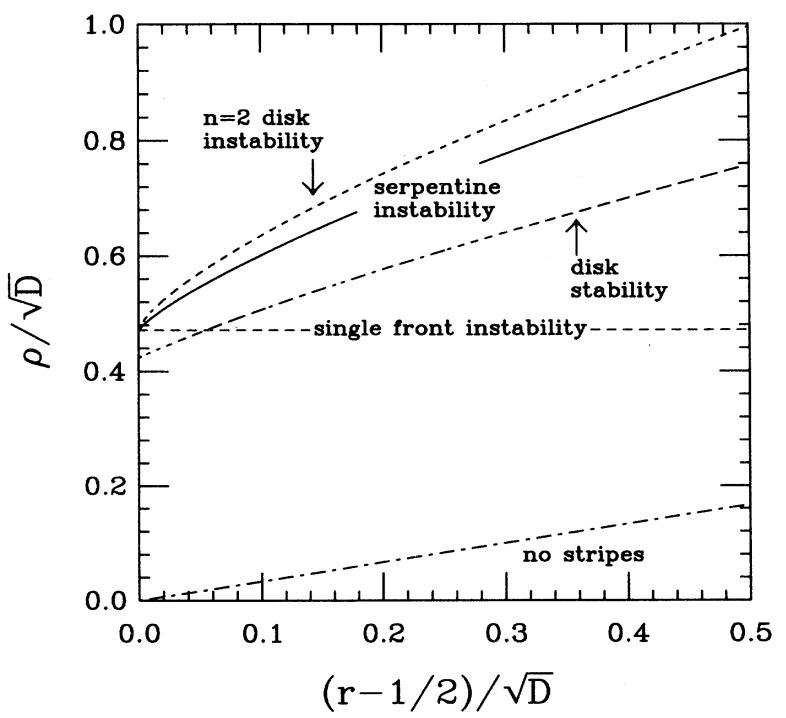

FIG. 12. Stability diagram for stripes, in the asymptotically rescaled parameter space. The coalescence of the sinuous instability of a stripe and the instability of a single front as $r \rightarrow \frac{1}{2}$ reflects the diverging equilibrium width of the stripe. Note that the sinuous instability lies in the region of azimuthally stable localized states. cation of the instability of a single front, as well as the boundary of stable stripes, defined by

$$
\tilde{\rho}=\frac{1}{3} \tilde{r} .
$$

Beyond the behavior of a single chemical front, it is natural to consider the stability of stripes. Perturbations to the shape of a stripe may be decomposed into those with odd and even parity under reflection through the stripe midline, termed sinuous $(\mathrm{S})$ and varicose $(\mathrm{V})$, respectively. In these two situations, the interfaces bounding a stripe of width $2 Q^{*}$ are parametrized as follows:

$$
\begin{aligned}
& \mathbf{r}_{ \pm}(x)=x \hat{\mathbf{e}}_{x}+\left[ \pm Q^{*}+\zeta(x, t)\right] \hat{\mathbf{e}}_{y} \quad(\mathrm{~S}) \\
& \mathbf{r}_{ \pm}(x)=x \hat{\mathbf{e}}_{x}+\left[ \pm Q^{*} \pm \zeta(x, t)\right] \hat{\mathbf{e}}_{y} \quad(\mathrm{~V})
\end{aligned}
$$

A straightforward calculation yields the growth rates of these two modes:

$$
\begin{aligned}
& \sigma_{\mathrm{S}}(k)=\sigma_{\mathrm{f}}(k)-3 \sqrt{2 D} \rho e^{-2 Q^{*}}\left(1-\frac{1}{f(k)} e^{-2 Q^{*}[f(k)-1]}\right), \\
& \sigma_{\mathrm{V}}(k)=\sigma_{\mathrm{f}}(k)-3 \sqrt{2 D} \rho e^{-2 Q^{*}}\left(1+\frac{1}{f(k)} e^{-2 Q^{*}[f(k)-1]}\right) .
\end{aligned}
$$

In each case, the growth rates are less than that of the single front. The sinuous mode grows faster than the varicose, whose damping at $k=0$ reflects the local stability of the preferred stripe width. This is consistent with what is known in other contexts, for instance in the buckling instability of magnetic stripes [18]. Figure 13 shows these growth rates for typical values of the parameters. The greater stability of a stripe relative to a single front may also be revealed by the onset of its sinuous instability at higher values of $\rho$. Expanding the growth rate (5.16a) to order $k^{4}$ as in Eq. (5.11), we obtain marginal stability conditions by setting the coefficient of $k^{2}$ to zero, yielding the transcendental equation

$$
\frac{\sqrt{2}}{3}=\tilde{\rho}\left\{1-\frac{\tilde{r}}{3 \tilde{\rho}}\left[1+\ln \left(\frac{3 \tilde{\rho}}{\tilde{r}}\right)\right]\right\}
$$

A numerical solution to this is shown in Fig. 12, where we see that this stability boundary properly merges with that of a single front as $r \rightarrow \frac{1}{2}^{+}$, and the equilibrium stripe width $Q^{*}$ diverges. Note that Eq. (5.17) is precisely the condition that the structural energy per unit length of the stripe (3.12) vanish. That these two conditions are related is seen by the fact that the coefficient of $k^{2}$ in the stability analysis is an effective line tension or energy per unit length. Beyond this stability boundary, the proliferation of stripes (straight or buckled) is energetically preferred. 


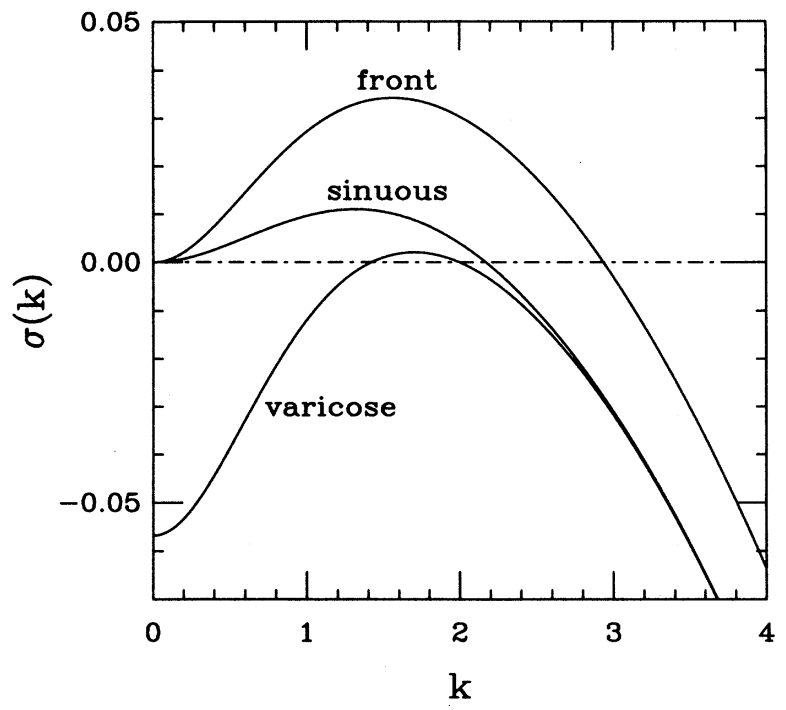

FIG. 13. Spectrum of growth rates for instabilities of isolated planar fronts, and the sinuous and varicose instabilities of stripes of finite width. Parameters are $D=0.01, \rho=0.3$, and $2 Q^{*}=1.5$. The single front is more unstable than the finite-width stripe, while the varicose mode is the most stable, particularly as $k \rightarrow 0$.

\section{Azimuthal instability of disks}

The evolution equation (4.33) that establishes the size of equilibrium disk solutions (4.34) also determines their nonlinear stability to radial perturbations. For azimuthal disturbances, the growth exponents are obtained by considering a disk whose radius develops a small sinusoidal variation

$$
r=R^{*}+\zeta(t) \sin n \theta
$$

The linearized curvature of the interface as calculated using (4.24) is

$$
\kappa \sim \frac{1}{R^{*}}+\left(n^{2}-1\right) \frac{\zeta(t)}{R^{* 2}} \sin n \theta,
$$

where it is noted that the $n= \pm 1$ perturbations are equivalent to simple translations of the disk. In this nearcircular geometry, it is advantageous to use the bulk approach to solve for the inhibitor field, since the leadingorder correction to the unperturbed inhibitor field involves only a single eigenmode. To $O(\zeta)$, the inhibitor field $v^{0}(r)$ is

$$
v_{n}^{0}(r, \theta) \sim v^{0}(r)+ \begin{cases}\zeta R^{*} K_{n}^{*} I_{n}(r) \sin n \theta, & r \leq R \\ \zeta R^{*} I_{n}^{*} K_{n}(r) \sin n \theta, & R \leq r\end{cases}
$$

where $v^{0}(r)$ is given in (4.32), $K_{n}^{*} \equiv K_{n}\left(R^{*}\right)$ and $I_{n}^{*}=$ $I_{n}\left(R^{*}\right)$, and the continuity conditions must now be imposed on the perturbed interface. This gives the corrected value of the inhibitor on the interface,

$$
v_{n}^{0}(\mathbf{r})=v_{r}^{0}\left(R^{*}\right)-\zeta R^{*}\left[K_{1}^{*} I_{1}^{*}-I_{n}^{*} K_{n}^{*}\right] \sin n \theta
$$

where the correction also vanishes for the $n= \pm 1$ modes.

Collecting the $O(\zeta)$ corrections from the front speed dynamics (4.28) yields the linearized growth exponent for the $n$ th-mode perturbation

$\sigma_{n}=6 \sqrt{2 D}\left\{\gamma \frac{\left(1-n^{2}\right)}{R^{* 2}}+\rho R^{*}\left[K_{1}^{*} I_{1}^{*}-I_{n}^{*} K_{n}^{*}\right]\right\}$.

The neutral curves for the lower mode instabilities acting on the disk solutions of region (a) are shown in Fig. 9. Coincidentally, note that the convergence point for these curves is identical to the critical $\rho_{c}(5.12)$ which is where $R^{*}$ is naturally large. The fact that the instabilities of disks and straight fronts derives from a common mechanism is demonstrated in the limit of large disks yet finite wave number $k=n / R^{*}$, yielding

$$
\lim _{n, R^{*} \rightarrow \infty} \sigma_{\left(k=n / R^{*}\right)}=\sigma_{f}(k)
$$

\section{NUMERICAL STUDIES}

\section{A. Simulation of PDEs}

In this section we present numerical studies of the reaction-diffusion dynamics $(2.1)$ to verify the stability results discussed in the preceding section, within the context of the parameter space of Fig. 12. In the subsequent section we investigate the contour dynamics for comparison. These numerical studies were performed using a pseudospectral algorithm in a two-dimensional periodic domain. The algorithm is outlined in the Appendix. In showing the time evolution, it is convenient to adopt a rescaled time $\tau \equiv \sqrt{2 D}(r-1 / 2) t$.

The first phenomenon illustrated is the twodimensional version of the approach in one dimension of two chemical fronts illustrated in Fig. 5. This is shown in Fig. 14 starting from an initial condition in which the two fronts are far apart and given a modulation by a random collection of Fourier modes. To indicate the symmetry of the underlying PDEs given in Eq. (2.5), the parameters chosen $(D=0.01, \rho=0.2, r=0.4)$ are such that the state $u=1$ is the more stable and invades the regions with $u=0$. The straight front is linearly stable, so in addition to the net motion of the fronts toward each other, these initial modulations relax. As in the onedimensional studies, for these parameter values the fronts do not cross, coming to rest at a finite distance set by the detuning $r-\frac{1}{2}$ and the inhibitor coupling $\rho$. Similar behavior has been seen in the iodide-ferrocyanide-sulfate reaction [1], as well as in simulations of the Grey-Scott model [2].

The growth of a labyrinthine structure from a compact initial condition was illustrated in Fig. 2. The parameter values for this computation, $\tilde{r}=0.2, \tilde{\rho}=1.5$, are in the region of rescaled parameter space in which the simple disk solution is unstable to many azimuthal modes. The black regions in the figure are those within which 


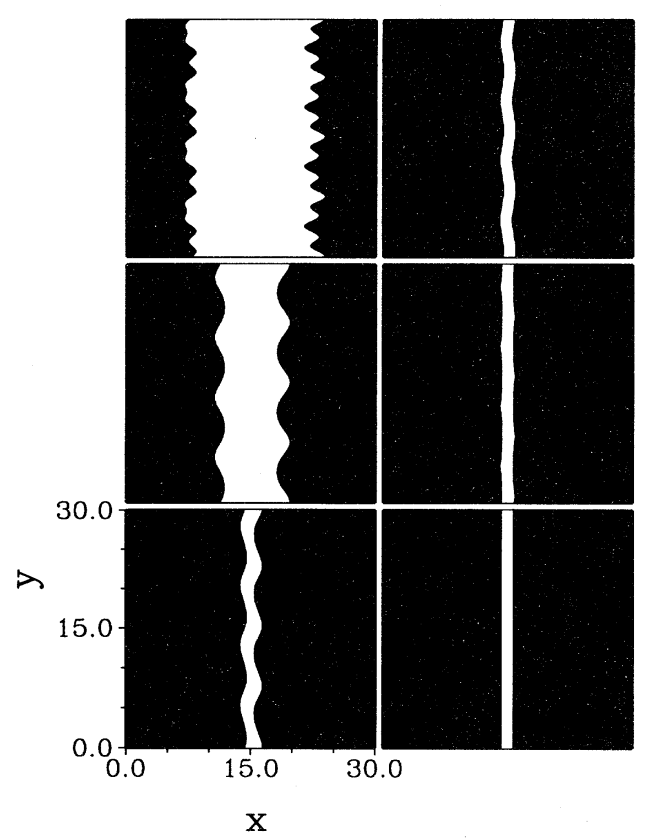

FIG. 14. Contour plots of the activator $u$ from numerical simulations of the reaction-diffusion model in two dimensions. Parameters are $D=0.01, r=0.40$, and $\rho=0.20$. In analogy with the one-dimensional front-repulsion phenomenon shown in Fig. 5, the approaching chemical fronts do not cross, and equilibrate to a simple stripe. Rescaled times are in increments of $\Delta \tau=6$ from upper left to lower right.

$u \geq \frac{1}{2}$. We see in the early stages of the evolution the growth of fingers of a well-defined width - this is the behavior discussed by Ohta, Mimura, and Kobayashi [8]. Several of the fingers undergo tip splitting. Their mutual repulsion leads eventually to a space-filling labyrinth that apparently converges to a steady state. This convergence is clearly a consequence of the front self-avoidance in the periodic computational domain. The interactions between the fronts have been such as to create a rather uniform width to the fingers of $u=1$, as well as to the intervening regions with $u=0$. Besides their similarity to the experimental patterns of Lee and co-workers $[1,2]$, the phenomenology of this pattern formation has a very strong resemblance to that seen in magnetic fluids [17] and superconductors [21], as well as thin garnet films $[22]$.

By changing the coefficients to $\tilde{r}=1, \tilde{\rho}=1$, we enter the regime in which the localized disk is stable, and a branched structure may relax to it without fissioning. This is shown in Fig. 3, in which the starting field configuration is panel (c) in Fig. 2. Again, this shape relaxation is like that seen in the iodide-ferrocyanide-sulfite reaction, as well as that observed in magnetic fluids in Hele-Shaw flow when the applied magnetic field is removed and surface tension returns a fingered structure to the circular ground state [20].

The front interactions responsible for labyrinthine fingering instabilities of a single domain naturally appear in the interactions of multiple domains, and can lead to

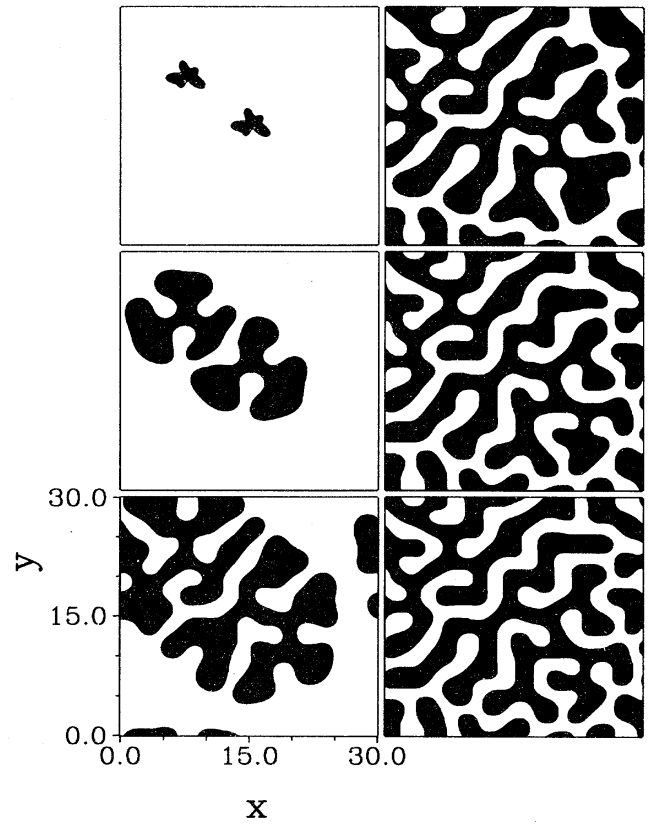

FIG. 15. Pattern formation with multiple domains of activator. $D=0.01, r=0.60, \rho=0.30, \Delta \tau=2.36$. The domains each undergo fingering instabilities, but do not merge.

patterns composed of disconnected but highly interdigitated regions. Figure 15 shows contour plots of the evolution starting from an initial condition with two small, nearly circular domains. Despite the complex fingering instabilities, the domains retain their integrity and do not merge. This multiple-domain problem is rather similar to that observed in the intermediate state of type-I superconductors (see also Sec. VIII).

An important aspect of the evolution of compact domains concerns the possibility of domain fission and fusion. As is quite common with interfacial treatments of pattern formation, the contour dynamics is not asymptotically valid when regions of the interface approach each other near the reconnection point. The full PDEs for the activator-inhibitor pair are, however, well defined during these events, and both fission and fusion are possible if the pressure driving the interfaces together is large enough or, conversely, the inhibitor-induced repulsion is small enough. This has also been observed in experiments [2].

\section{B. Simulation of contour dynamics}

Next we turn to numerical studies of the nonlocal contour dynamics. The numerical method for this, outlined in the Appendix, has been employed elsewhere [43] for analogous problems in nonlocal interface motion. It is based on a pseudospectral treatment of the tangent-angle representation of the dynamics.

The most elementary instability of a localized state is the elliptical one, illustrated in Fig. 16. Here, the initial condition is a solution of the equilibrium condition (4.34) 

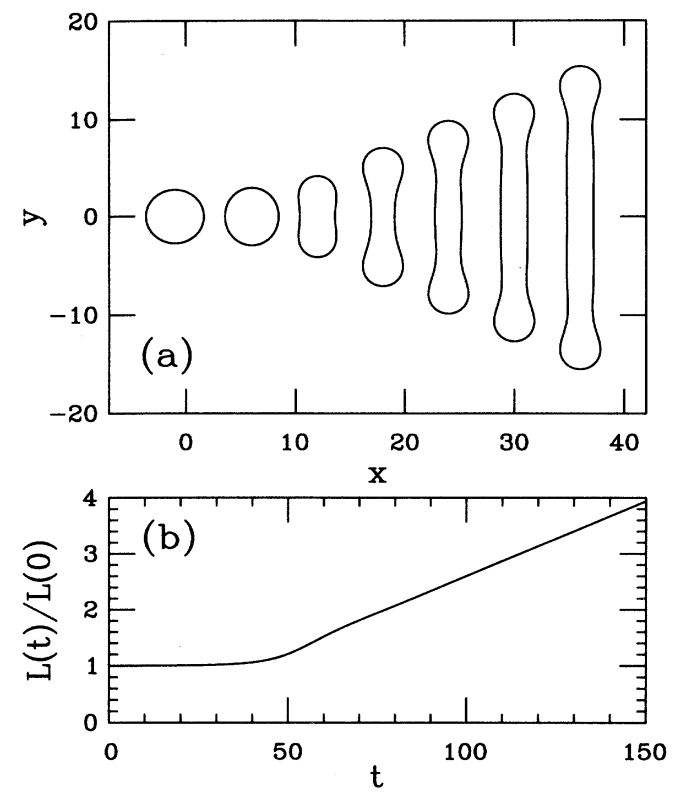

FIG. 16. Numerical simulation of contour dynamics showing destabilization of a circle. Lower panel shows perimeter as a function of time, illustrating the asymptotically linear growth. Parameters are $\tilde{r}=0.212, \tilde{\rho}=0.796$, with $\Delta \tau=25$.

for a localized disk, perturbed with a small amplitude of the $n=2$ mode. After a short transient, the domain enters an era of linear elongation as illustrated in the lower panel of the figure. This linear growth is a reflection of the negative structural energy density (3.12) for the parameters $\tilde{r}=0.212, \tilde{\rho}=0.796$.

Increasing the value of the inhibitor coupling to $\rho=$ 0.90 renders the localized state unstable to the mode $n=3$ (as well as to the elliptical mode). The bulbous tips that form during the elliptical instability have a nonzero projection onto the $n=3$ mode, leading to tip splitting events. This is shown in Fig. 17. In this regime of

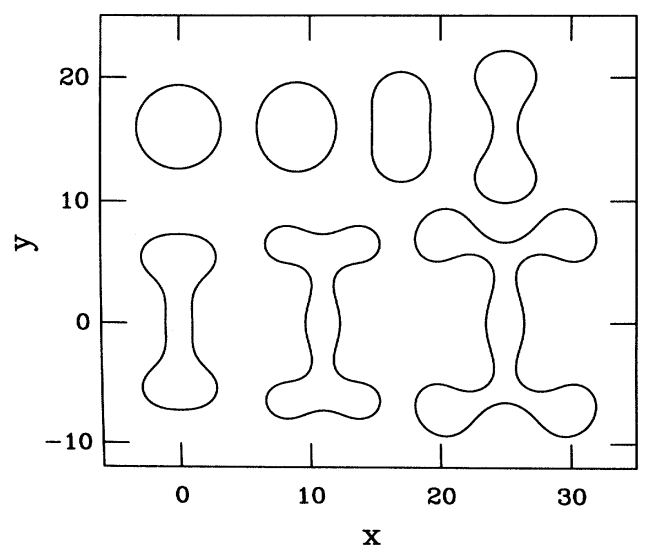

FIG. 17. Tip splitting in the contour dynamics. Initial condition is a localized state perturbed by a small $n=2$ distortion. Parameters are $\tilde{r}=0.212, \tilde{\rho}=0.90, \Delta \tau=10$; both the $n=2$ and $n=3$ modes are unstable.

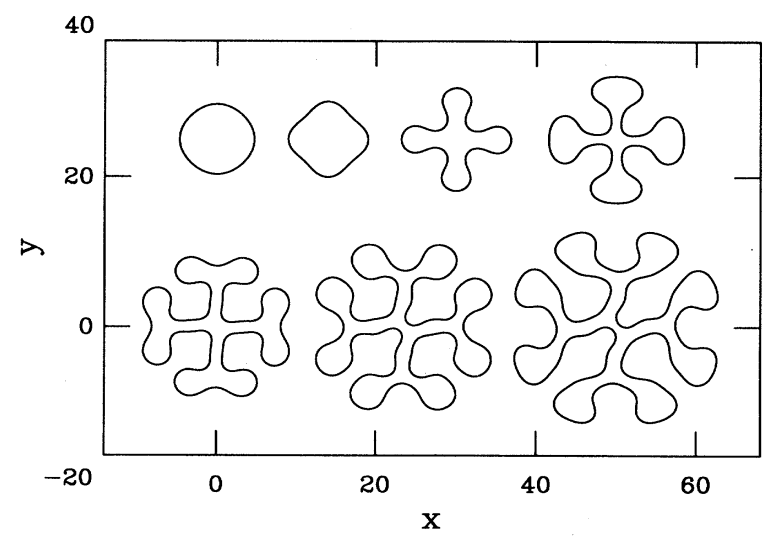

FIG. 18. Instability of a fourfold vertex. Contour dynamics results, the initial condition being a fourfold perturbed localized state with an additional very small $n=2$ distortion. $\tilde{r}=0.212, \tilde{\rho}=1.10, \Delta \tau=5$.

parameter space, each time a new tip is formed it is susceptible to tip splitting, leading to a cascading process and a proliferation of threefold coordinate vertices.

For $\rho$ larger still, we may investigate one of the common features of labyrinthine structures seen in dipolar systems as well as in chemical reactions: the appearance of threefold nodes to the exclusion of all others of higher coordination. For certain kinds of optimization problems involving the minimization of interface length under the constraint of fixed endpoints, it is known that threefold nodes are the only stable vertices [50], but no such result is known in the present context. The numerical simulations strongly suggest, however, that higher-order vertices are dynamically unstable. Figure 18 shows the evolution of an initial condition consisting of a localized disk modulated by fourfold perturbations of several percent and a twofold distortion one fiftieth as large. A crosslike vertex forms quickly, but is unstable to the elliptical perturbation, splitting into two threefold vertices.

Having seen in isolation the elementary processes underlying labyrinth formation, stripe formation and proliferation, tip splitting, and vertex reduction, we show in Fig. 19 the appearance of a labyrinthine pattern from a compact initial condition. The characteristic feature of interface repulsion and the appearance of well-defined fin-. ger widths is readily apparent. Unlike the simulations of the PDEs, the contour representation does not naturally build in periodic boundary conditions, so the long-time evolution of the two will differ significantly. Changing the coefficients so that the localized disk is both radially and azimuthally stable, we see in Fig. 20 the relaxation to a compact state of a branched initial condition taken from near the the end of the evolution in Fig. 19.

\section{BEYOND THE SLAVING LIMIT}

As we have discussed so far, the limit $\epsilon \rightarrow 0$ renders the dynamics an overdamped gradient flow, associated with a Lagrangian variational principle in which the kinetic 


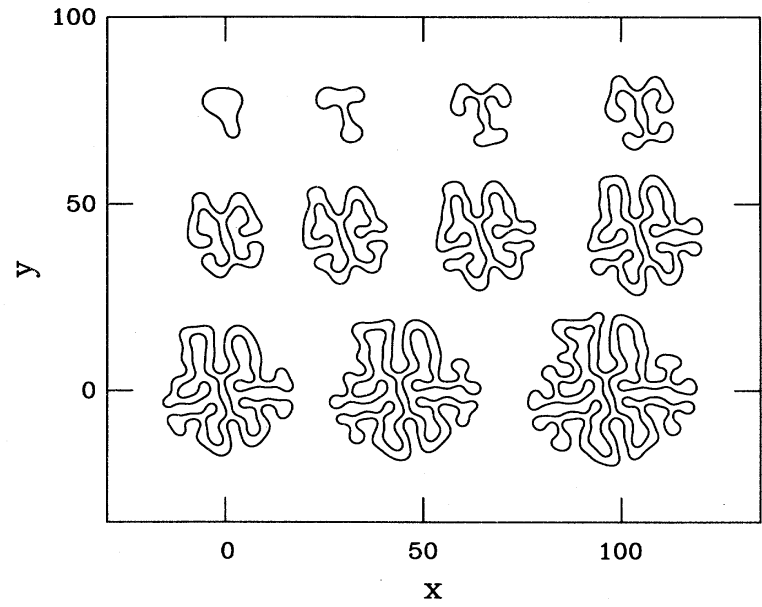

FIG. 19. Growth of a labyrinth from a compact initial condition. Contour dynamics results proceed in time from upper left to lower right, spaced by $\Delta \tau=5$. Parameters are $\tilde{r}=0.21, \tilde{\rho}=1.10$, and initial condition is a distorted circle of radius $R=6.0$.

energy is neglected and the dissipation function is local. As the recent work of Hagberg and Meron [26,27] has emphasized, the nature of the chemical fronts between the two metastable values of the activator may be quite different in the two extremes of slow and fast inhibition. From Eq. (4.28), the time scale of the front dynamics is $u_{t}=O(D)$, so that in actuality, the fast-inhibitor limit only requires that $\epsilon D \ll 1$ - so that the value of $\epsilon$ can be quite large. Extending this contour dynamic approach beyond this fast-inhibitor limit represents a significant challenge. Nevertheless, an intuition for how oscillatory behavior might arise with finite $\epsilon$ is suggested by arguments using contour energetics.

For $\epsilon \neq 0$ we may solve the inhibitor dynamics $(2.4 \mathrm{~b})$ for $v$ in terms of $u$,

$$
v(\mathbf{x}, t)=\int^{t} d t^{\prime} \int d \mathbf{x}^{\prime} G\left(\mathbf{x}-\mathbf{x}^{\prime}, t-t^{\prime}\right) u\left(\mathbf{x}^{\prime}, t^{\prime}\right)
$$

where for $t>0$ the Green's function is

$$
G(\mathbf{x}, t)=\frac{1}{4 \pi t} \exp \left\{-\frac{t}{\epsilon}-\frac{\epsilon \mathbf{x}^{2}}{4 t}\right\} .
$$

Clearly $\epsilon$ is the natural time scale for the decay of $G$, and if we take the liberty of expanding the integral for slowly varying $u$ we obtain $u\left(\mathbf{x}^{\prime}, t^{\prime}\right) \simeq u\left(\mathbf{x}^{\prime}, t\right)+\left(t^{\prime}-\right.$ t) $u_{t}\left(\mathbf{x}^{\prime}, t\right)+\left(\frac{1}{2}\right)\left(t^{\prime}-t\right)^{2} u_{t t}\left(\mathbf{x}^{\prime}, t\right)+\cdots$, substitute into (7.2) and perform the time integrations. Each power of $t^{\prime}-t$ in the expansion will contribute a power of $\epsilon$. Up to order $\epsilon^{2}$ we obtain

$$
\begin{aligned}
v(\mathbf{x}, t) \simeq & \int d \mathbf{x}^{\prime}\left\{\mathcal{G}_{0}\left(\mathbf{x}-\mathbf{x}^{\prime}\right) u\left(\mathbf{x}^{\prime}, t\right)\right. \\
& +\epsilon \mathcal{G}_{1}\left(\mathbf{x}-\mathbf{x}^{\prime}\right) u_{t}\left(\mathbf{x}^{\prime}, t\right) \\
& \left.+\epsilon^{2} \mathcal{G}_{2}\left(\mathbf{x}-\mathbf{x}^{\prime}\right) u_{t t}\left(\mathbf{x}^{\prime}, t\right)\right\}
\end{aligned}
$$

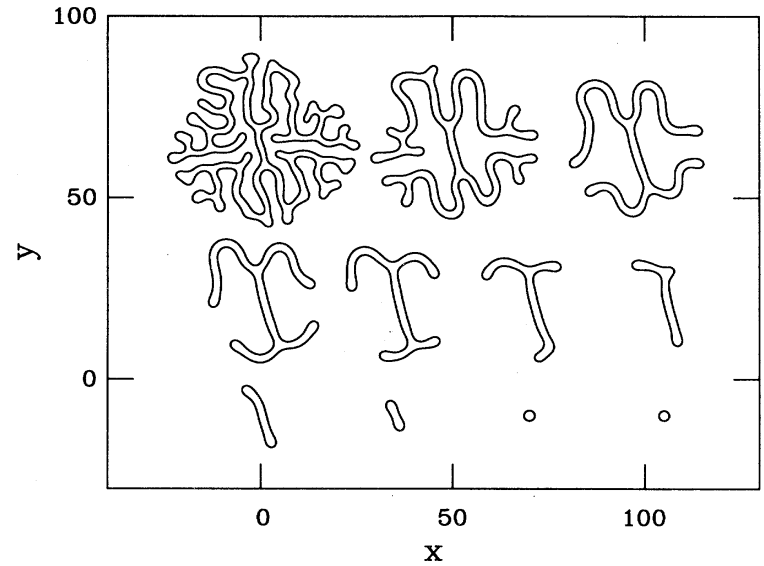

FIG. 20. Relaxation of a labyrinthine pattern to a localized state. Initial condition is close to the final panel in Fig. 19, with parameters $\tilde{r}=0.21, \tilde{\rho}=0.601$, and time between figures of $\Delta \tau=25$.

where $\mathcal{G}_{0}(\mathbf{x})=(1 / 2 \pi) K_{0}(x)$ and the remaining functions $G_{i}$ are

$$
\mathcal{G}_{1}(\mathbf{x})=-\frac{1}{4 \pi} x K_{1}(x), \quad \mathcal{G}_{2}(\mathbf{x})=\frac{1}{16 \pi} x^{2} K_{2}(x) .
$$

Note the very important feature that the order $\epsilon$ kernel $\mathcal{G}_{1}$ is negative, while the second-order kernel is positive. Up to quadratic order in $\epsilon$ the nonlocal activator dynamics may be written in the following form:

$$
\begin{aligned}
\epsilon^{2} \rho & \int d \mathbf{x}^{\prime} \mathcal{G}_{2}\left(\mathbf{x}-\mathbf{x}^{\prime}\right) u_{t t}\left(\mathbf{x}^{\prime}, t\right)+\frac{\delta \mathcal{E}}{\delta u} \\
& =-u_{t}-\epsilon \rho \int d \mathbf{x}^{\prime} \mathcal{G}_{1}\left(\mathbf{x}-\mathbf{x}^{\prime}\right) u_{t}\left(\mathbf{x}^{\prime}, t\right)
\end{aligned}
$$

This conforms to the variational principle in Eq. (3.7), having the form

$$
\partial_{t} \frac{\delta \mathcal{T}}{\delta u_{t}}+\frac{\delta \mathcal{E}}{\delta u}=-\frac{\delta \mathcal{R}}{\delta u_{t}},
$$

but now with a finite kinetic energy functional

$$
\mathcal{T}=\frac{1}{2} \epsilon^{2} \rho \int d \mathbf{x} \int d \mathbf{x}^{\prime} u_{t}(\mathbf{x}, t) \mathcal{G}_{2}\left(\mathbf{x}-\mathbf{x}^{\prime}\right) u_{t}\left(\mathbf{x}^{\prime}, t\right)
$$

and a nonlocal contribution to the dissipation function

$$
\begin{aligned}
\mathcal{R}= & \frac{1}{2} \int d \mathbf{x} u_{t}^{2}(\mathbf{x}, t) \\
& +\frac{\epsilon \rho}{2} \int d \mathbf{x} \int d \mathbf{x}^{\prime} u_{t}(\mathbf{x}, t) \mathcal{G}_{1}\left(\mathbf{x}-\mathbf{x}^{\prime}\right) u_{t}\left(\mathbf{x}^{\prime}, t\right) .
\end{aligned}
$$

The positivity of $\mathcal{G}_{2}$ renders the "mass" of the field dynamics positive, while there is a competition between the signs of the local and nonlocal parts of the dissipation function.

A heuristic derivation of the leading-order changes to the contour dynamics from the inclusion of nonzero $\epsilon$ proceeds as in Sec. IV. Starting from the dynamics for $u$ in 
(7.5) or equivalently the functionals in (7.7) and (7.8), we recognize that the time derivative $u_{t}$ and acceleration $u_{t t}$ are localized at the domain boundary. Using a correspondence like that in Eq. (3.9), we obtain the contour representation of the kinetic energy,

$$
\mathcal{T} \simeq \frac{\rho \gamma^{2} \epsilon^{2}}{2 D} \oint d s \oint d s^{\prime} \hat{\mathbf{n}} \cdot \mathbf{r}_{t} \mathcal{G}_{2}\left(\mathbf{r}-\mathbf{r}^{\prime}\right) \hat{\mathbf{n}} \cdot \mathbf{r}_{t}^{\prime},
$$

and dissipation function

$$
\begin{aligned}
\mathcal{R} \simeq & \frac{\gamma}{2 D} \oint d s\left(\hat{\mathbf{n}} \cdot \mathbf{r}_{t}\right)^{2} \\
& +\frac{\gamma^{2} \rho \epsilon}{2 D^{2}} \oint d s \oint d s^{\prime} \hat{\mathbf{n}} \cdot \mathbf{r}_{t} \mathcal{G}_{1}\left(\mathbf{r}-\mathbf{r}^{\prime}\right) \hat{\mathbf{n}} \cdot \mathbf{r}_{t}^{\prime}
\end{aligned}
$$

This kind of nonlocal dissipation function occurs as well in the analysis of certain models of solidification [51,52] and is conceptually similar to the role of the Oseen tensor in polymer dynamics [53].

The interpretation of this dynamics as a damped mechanical system is complicated by the nonlocal nature of the kinetic energy and dissipation functionals. With the function $\mathcal{G}_{1}<0$, the overall rate of dissipation is positive for small $\epsilon$ but may become negative for $\epsilon$ sufficiently large, say of order $1 / \rho$. One expects qualitatively new behavior in this limit. Moreover, the inclusion of inertial terms in the dynamics makes the analysis of travelingwave states qualitatively different from that in the overdamped limit. Specifically, an ansatz of the form $u(x-c t)$ yields a cubic equation for the speed $c$, with three possible roots, in contrast to the unique speed found in the limit $\epsilon \rightarrow 0$. These two roots most likely represent those associated with the nonequilibrium Ising-Bloch front bifurcation discussed by Hagberg and Meron [27], and are related to the oscillatory instabilities discussed by Ohta, Mimura, and Kobayashi [8] for the case of simple geometries.

Developing a theory for the asymptotic stability of the labyrinthine patterns is one current effort [54]. It is hoped that results in this direction will lead to a quantitative connection between the problem of finite inhibitor diffusion $(\epsilon \neq 0)$ and the onset of time-oscillatory behavior.

\section{NONLOCAL CONTOUR DYNAMICS IN OTHER SYSTEMS}

We have focused here on the fast-inhibitor limit of a reaction-diffusion system and demonstrated that its behavior is well described by a nonlocal contour dynamics model. In this limit, where the inhibitor is slaved to the activator, the dynamics is a gradient flow with an energy functional of the form

$$
\mathcal{E}[\mathbf{r}]=\Pi A+\gamma L-\frac{1}{2} \rho \oint d s \oint d s^{\prime} \hat{\mathbf{t}} \cdot \hat{\mathbf{t}}^{\prime} \Phi(R / h),
$$

with $R=\left|\mathbf{r}(s)-\mathbf{r}\left(s^{\prime}\right)\right|$, and a normal velocity $U$ proportional to the force obtained variationally as $-\hat{\mathbf{n}} \cdot \delta \mathcal{E} / \delta \mathbf{r}$, with

$$
U=-\Pi-\gamma \kappa+\frac{\rho}{h} \oint d s^{\prime} \hat{\mathbf{R}}\left(s, s^{\prime}\right) \times \hat{\mathbf{t}}^{\prime} \Phi^{\prime}(R / h) .
$$

In this section we make specific the connections between this form of dynamics and those found in several other quite distinct physical systems. These are (i) type-I superconductors in the intermediate state [55], (ii) magnetic fluids in Hele-Shaw flow $[17,20,39,42,56]$, and (iii) Langmuir monolayers of dipolar molecules [43,57]. While the functions $\Phi$ differ from one system to the next, the common feature we find in each is a positive bare line tension $\gamma$, and a repulsive interaction between antiparallel tangent vectors, with $\rho>0$. Interfacial instabilities leading to fingered structures are then seen to derive from a negative effective line tension arising from the nonlocal contribution. It is this same nonlocal coupling of the interface that leads to the self-avoiding nature of the pattern formation beyond the linear instability. We discuss each of the three systems below, pointing out the different physical origins of the nonlocal coupling, and the different constraints on the interface motion.

Type-I superconductors. The intermediate state of type-I superconductors occurs when a thin slab of the material below its zero-field transition temperature is placed in a magnetic field normal to its surface [21]. Rather than exhibit a complete Meissner effect, the demagnetizing effects arising from the sample geometry lead instead to the penetration of the flux through the sample in an intricate arrangement of flux domains, each of which is fingered and often branched. The shapes of these flux domains arise from a competition between the positive superconductor-normal surface energy and the interactions between the Meissner currents which circulate at the flux domain boundaries. As argued many years ago $[58,59]$, this self-induction interaction retains its long-range form as in free space, since the electromagnetic fields in the vacuum above and below the slab are unscreened. In the simplest treatment of these interactions [55], they are considered identical to those of current loops in completely free space. Upon averaging the standard Coulombic self-interaction between elementary current segments over the thickness $h$ of the slab, one finds a tangent-vector coupling for a single interface

$$
\begin{aligned}
\Phi(z) & =-\ln \left[z^{-1}+\left(1+z^{-2}\right)^{1 / 2}\right]+z-\left[1+z^{2}\right]^{1 / 2} \\
-\Phi^{\prime}(z) & =\left[1+z^{-2}\right]^{1 / 2}-1
\end{aligned}
$$

where the characteristic length scale is $h$. While retaining the Coulombic form $\Phi(z) \sim 1 / z$ for $z \rightarrow \infty, \Phi$ has only a logarithmic singularity at the origin, much like the Bessel function $K_{0}$ in the reaction-diffusion problem considered here.

Interface motion in superconducting systems does not conform to the simple local-dissipation model discussed in Sec. IV, but rather reflects the diffusion of the magnetic flux in the normal phase [60]. Thus, the force (8.2) becomes a boundary condition for the diffusion equation obeyed by the field, rather than determining the velocity directly [38]. In simple models of this pattern formation [55], in which the local dissipation model is invoked, this conservation law leads to the generalization of the product II $A$ in Eq. (8.1) to the derivative of a bulk free energy density $\mathcal{E}_{\text {bulk }}(A)$, which arises from the competi- 
tion between the field energy of the external magnetic field and the condensation free energy of the superconducting state. The conservation of magnetic flux is an important global constraint in this problem, and leads to an equilibrium area fraction of the domains that is determined primarily by the minimization of this energy; the shapes of the individual interfaces arise from the competing line tension and Biot-Savart interactions. Unlike the reaction-diffusion problem, the intermediate state properties are determined fundamentally by many-domain interactions.

Magnetic fluids. A second physical system conforming to the energetics in Eqs. (8.1) and (8.2) is that of thin domains of magnetic fluids [17] in the geometry of Hele-Shaw flow. There, the domain is trapped between two glass plates spaced a distance $h$ apart, with the remainder of the gap filled by water. A magnetic field applied normal to the plates aligns the microscopic magnetic domain in suspension, creating an approximately uniformly magnetized droplet. By the correspondence between magnetization and current loops, the field energy associated with the domain is again represented in terms of a self-induction interaction, with the functions $\Phi$ and $\Psi$ as in (8.3) by virtue of the slab geometry. The amplitude $\rho$ is now proportional to $M^{2} h$, where $M$ is the magnetization of the domain, and the line tension $\gamma=h \sigma$, with $\sigma$ the ferrofluid-water surface tension.

In this hydrodynamic problem, the dynamics is wellapproximated by Darcy's law $\mathbf{v}=-\left(h^{2} / 12 \eta\right) \nabla P$, where $\mathbf{v}$ is the $z$-averaged in-plane fluid velocity, $\eta$ the fluid viscosity, and $P$ a generalized pressure including magnetic contributions $[20,42]$. With the constraint of fluid incompressibility, the pressure field is harmonic, with the force (8.2) again acting as a boundary condition on $P$. The fluid incompressibility leads directly to the conservation of the area enclosed by the boundary.

Langmuir monolayers. The final class of systems governed by these energetics includes amphiphilic (Langmuir) monolayers at the air-water interface. These consist of single- or multiple-component monomolecular films of surfactants in which the mean lateral density is regulated externally, allowing for a study of phases and phase transformations. Under suitable conditions, domains of a high-density phase appear in a background of lower density and may be visualized by the differential fluorescence of a dye incorporated into the layer. One observes various shape instabilities of these domains as conditions such as temperature and pressure are varied [16]. These are believed to arise from the competing effects of line tension at the domain boundary and longrange electric dipole interactions between the molecules which are oriented by the constraint of packing. The energetics of these interactions may be described [43] by taking the "ultrathin" limit of the magnetic formulation (8.3) above, with the cutoff $h$ being a molecular length, and the amplitude $\rho$ proportional to the square of the dipole moment density $\mu$. In the limit of small $h$, the self-induction and Biot-Savart terms have the form associated with infinitesimal current-carrying wires [57],

$$
\Phi(z)=\frac{1}{z}, \quad-\Phi^{\prime}(z)=\frac{1}{z^{3}} .
$$

A cutoff procedure must be implemented on these functions to treat the divergences that occur when $s-s^{\prime} \rightarrow 0$. As in the superconductor problem, in the simplest model [43], $\Pi$ is a Lagrange multiplier conjugate to the area. Experiments [61] have shown a variety of fingered domain shapes consistent with the boundary model. More recent work has focused on the hydrodynamics of monolayer domains coupled to the viscous subfluid $[62,63]$.

The nonlocal energy functional $\mathcal{E}[u]$ in Eq. (2.18) is known also to be relevant for physical systems quite distinct from those with dipolar interactions, appearing, for instance, in models of microphase separation in block copolymers [64]. There the nonlocal coupling is long-ranged, reflecting the connectivity of the polymers. Labyrinthine patterns occur there as well (see also the review in Ref. [23]), but are not necessarily confined to two dimensions. It has also been remarked [65] that this nonlocal interface coupling may be related to the diffusion of impurities and/or latent heat in solidification, which leads to some degree of interface self-avoidance seen in the development of dendrites. Such behavior cannot be captured by purely local geometric [49] or boundary-layer [66] models. Finally, observe that the Biot-Savart coupling also appears in the contour dynamics formulation [67-69] of vortex patch motion in two-dimensional ideal fluids. Rather than exhibiting strongly overdamped dynamics, these are of course Hamiltonian systems.

\section{CONCLUSIONS}

Finally, we discuss briefly three important open issues regarding the dynamics of labyrinthine pattern formation: generalizations to higher spatial dimension, further elucidation of variational principles, and derivation from microscopic chemical kinetics.

We have seen that the phenomenon of lateral inhibition necessarily involves interactions between segments of chemical fronts that are potentially far apart in arclength, yet close in space. It is precisely this nonlocality that enters the energetics (8.1) and dynamics (8.2). Whereas in the magnetic systems the angular part of the current-current interactions is naturally written as $\hat{\mathbf{t}}(s) \cdot \hat{\mathbf{t}}\left(s^{\prime}\right)$ in accord with the existence of currents circulating in the direction $\hat{\mathbf{t}}$, there is no such circulation in the reaction-diffusion problem. But of course, as seen in the derivation (4.3), this scalar product may equally well be written in terms of the normal vectors as $\hat{\mathbf{n}}(s) \cdot \hat{\mathbf{n}}\left(s^{\prime}\right)$. This formulation makes it clear that the central issue is whether or not fronts oppose one another with a region of low activator concentration in between. The normal vector representation allows a straightforward generalization to the interaction of two-dimensional surfaces, for which there is no natural or unique assignment of tangent vectors. We suggest that certain three-dimensional patterns may be profitably studied by models embodying this nonlocal interaction. In addition to the block copolymer systems mentioned earlier, other candidates include highly convoluted structures such as brain coral, which displays labyrinthine structures with features such as threefold coordinated nodes like those seen in the present work. 
One may imagine that these arise from the interplay between growth of individual members of the colony and the competition for nutrients.

As discussed in Sec. VII, a heuristically derived contour dynamics for small deviations from the fast-inhibitor limit appears to conform to a rather general variational principle much like that of a damped mechanical system. An issue of some significance is the extent to which the contour dynamics approach may be more rigorously extended to incorporate the front bifurcation that ultimately occurs for $\epsilon$ sufficiently large. Related issues concern the connection between such a description and spiral-wave behavior, as well as the nature of the front bifurcation for surfaces moving in three dimensions.

In light of the present derivation of the contour dynamics from the FitzHugh-Nagumo model, it remains of great interest to investigate as well whether the particular chemical kinetics [70] relevant to the experiments of Lee and co-workers $[1,2]$ may be recast as an interface dynamics. Recent work [71] has shown that those very complex kinetics have dynamics on many time scales and may be reduced to an effective two-variable model through a sequence of slaving approximations. While the form of that reduced description is somewhat different than the FitzHugh-Nagumo model, the possibility that it shows similar, near-gradient-flow behavior is an intriguing area of investigation.

\section{ACKNOWLEDGMENTS}

We are indebted to K.J. Lee, W.D. McCormick, Q. Ouyang, and H.L. Swinney for extensive discussions concerning their experimental work and for Fig. 1; to A.J. Bernoff, A.T. Dorsey, and K.J. Lee for detailed comments on the manuscript; and to D. Levermore for important suggestions at an early stage of this work. D.J.M. thanks Y. Kodama for introducing him to the Lie transform. We have also benefited from discussions with S. Erramilli, E. Knobloch, S. Leibler, E. Meron, M.J. Shelley, and V. Hakim. This work has been supported by NSF Grant No. DMR-9350227 and the Alfred P. Sloan Foundation (REG), NSF Grant No. DMS-9404374, and DOE Grant No. DE-FG02-88ER25053 (D.J.M.).

\section{APPENDIX: NUMERICAL METHODS}

Here we summarize established pseudospectral methods $[72,73]$ that we have adapted to study both the reaction-diffusion dynamics and the contour evolution. For the case of a scalar partial differential equation in $1+1$ dimensions,

$$
\frac{\partial u}{\partial t}=\mathcal{L}\left(\partial_{x}\right) u+\mathcal{N}[u]
$$

where $\mathcal{L}$ is a linear operator and $\mathcal{N}$ is nonlinear. We assume that the highest-order spatial derivative appears in the linear operator. In Fourier space (A1) is

$$
\frac{\partial \hat{u}(k, t)}{\partial t}-\omega(k) \hat{u}(k, t)=\hat{\mathcal{N}}(k, t)
$$

with $\omega(k)=\mathcal{L}(i k)$. The nonlinear terms are obtained pseudospectrally by fast Fourier transforming $\mathcal{N}$ in real space, $\hat{\mathcal{N}}(k, t)=\mathcal{F}[\mathcal{N}(u(x, t))]$, and $u(x, t)$ is computed by an inverse fast Fourier transformation of $\hat{u}(k, t)$. Now define

$$
\hat{v}(k, t)=e^{-\omega(k) t} \hat{u}(k, t),
$$

and multiply (A2) by the exponential factor, yielding

$$
\frac{\partial \hat{v}(k, t)}{\partial t}=e^{-\omega(k) t} \hat{\mathcal{N}}\left[\hat{v} e^{\omega(k) t}\right] .
$$

In the simplest Euler method, the solution to Eq. (A4) is

$$
\frac{\hat{v}(k, t+\Delta t)-\hat{v}(k, t)}{\Delta t} \simeq e^{-\omega(k) t} \hat{\mathcal{N}}\left(\hat{v}(k, t) e^{\omega(k) t}\right)
$$

which yields

$$
\hat{u}(k, t+\Delta t)=e^{\omega(k) \Delta t}[\hat{u}(k, t)+\Delta t \hat{\mathcal{N}}(k, t)] .
$$

The exponentiation of the growth rate $\omega(k)$ in (A6) plays a useful role in guaranteeing stability for a diffusive linear operator $\left[\omega(k)=-\gamma k^{2}\right]$. The usual stability considerations [74] would require a time step $\Delta t$ such that for large values of momentum (near the Brillouin zone edge $k_{\max }=\pi / a$, with $a$ the lattice spacing in real space) the quantity $k^{2} \Delta t$ be less than unity. This requires an extremely small time step, rendering the calculation prohibitively slow. Here, even if $k_{\max }^{2} \Delta t>1$, the calculation is stable due to the incorporation of the exact dynamics of the linear operator, namely the exponential damping at high momentum.

The generalization of Eq. (A6) to a fourth-order Runge-Kutta method proceeds as follows. Define

$$
L_{1 / 2}(k)=e^{\omega(k) \Delta t / 2}, \quad L(k)=e^{\omega(k) \Delta t},
$$

and the intermediate results

$$
\begin{aligned}
& \hat{U}_{1}(k)=L_{1 / 2}(k)\left[\hat{u}+\frac{1}{2} \Delta t \hat{\mathcal{N}}(\hat{u})\right] \\
& \hat{U}_{2}(k)=L_{1 / 2}(k) \hat{u}+\frac{1}{2} \Delta t \hat{\mathcal{N}}_{1}, \\
& \hat{U}_{3}(k)=L(k) \hat{u}+\Delta t L_{1 / 2}(k) \hat{\mathcal{N}}_{2},
\end{aligned}
$$

with $\hat{\mathcal{N}}_{j}=\hat{\mathcal{N}}\left(\hat{U}_{j}\right)$. Then the time-stepping routine analogous to (A6) is

$$
\begin{aligned}
\hat{u}(t+\Delta t)= & L(k) \hat{u}+\frac{1}{6} \Delta t L(k) \hat{\mathcal{N}}+\frac{1}{3} \Delta t L_{1 / 2}(k) \hat{\mathcal{N}}_{1} \\
& +\frac{1}{3} \Delta t L_{1 / 2}(k) \hat{\mathcal{N}}_{2}+\frac{1}{6} \Delta t \hat{\mathcal{N}}_{3} .
\end{aligned}
$$

To generalize this method to situations with $n$ coupled variables $u_{i}(i=1,2, \ldots, n)$, we write the equation of motion in Fourier space in vectorial form

$$
\hat{\mathbf{u}}_{t}=\boldsymbol{\Omega} \cdot \hat{\mathbf{u}}+\hat{\mathcal{N}}
$$

with $\boldsymbol{\Omega}$ a matrix of wave-vector-dependent growth rates in Fourier space and $\hat{\mathcal{N}}$ a vector of nonlinear terms obtained pseudospectrally. Now suppose that $\operatorname{det} \boldsymbol{\Omega}-\boldsymbol{\omega} \mathbf{I}=$ 
0 has as solutions eigenvalues $\omega_{i}$ and associated eigenvectors $\hat{\mathbf{e}}_{i}(i=1, \ldots, n)$. The matrix $\mathbf{T}$ whose columns are the components of the eigenvectors defines a linear transformation between $\mathbf{u}$ and the vector $\mathbf{a}$ of expansion coefficients in the basis of eigenvectors. That is, $\hat{\mathbf{u}}=\mathbf{T} \cdot \mathbf{a}$, where $\mathbf{T}^{-1} \cdot \mathbf{T}=\mathbf{I}$. The equation of motion (A12) then becomes

$$
\mathbf{a}_{t}=\mathbf{T}^{-1} \cdot \mathbf{\Omega} \cdot \mathbf{T} \cdot \mathbf{a}+\mathbf{T}^{-1} \cdot \hat{\mathcal{N}}
$$

The matrix $\mathbf{D} \equiv \mathbf{T}^{-1} \cdot \boldsymbol{\Omega} \cdot \mathbf{T}$ is diagonal. Now let $\mathbf{v} \equiv$ $\exp (-\mathbf{D} t) \mathbf{a}$, so

$$
\mathbf{v}_{t}=e^{-\mathbf{D} t} \mathbf{T}^{-1} \cdot \hat{\mathcal{N}}
$$

It follows that for a Euler method, one need only know the matrix

$$
\mathbf{L} \equiv \mathbf{T} \cdot e^{\mathbf{D} \Delta t} \mathbf{T}^{-1}
$$

for then

$$
\hat{\mathbf{u}}(k, t+\Delta t)=\mathbf{L} \cdot[\hat{\mathbf{u}}(k, t)+\Delta t \hat{\mathcal{N}}(k, t)],
$$

whereas in the Runge-Kutta method we obtain the vectorial analog of Eq. (A11),

$$
\begin{aligned}
\hat{\mathbf{u}}(k, t+\Delta t)= & \mathbf{L} \cdot \hat{\mathbf{u}}(k, t)+\frac{1}{6} \Delta t \mathbf{L} \cdot \hat{\mathcal{N}}+\frac{1}{3} \Delta t \mathbf{L}_{1 / 2} \cdot \hat{\mathcal{N}}_{1} \\
& +\frac{1}{3} \Delta t \mathbf{L}_{1 / 2} \cdot \hat{\mathcal{N}}_{2}+\frac{1}{6} \Delta t \hat{\mathcal{N}}_{3},
\end{aligned}
$$

where

$$
\mathbf{L}_{1 / 2} \equiv \mathbf{T} \cdot e^{\mathbf{D} \Delta t / 2} \mathbf{T}^{-1}
$$

and the intermediate results are

$$
\begin{aligned}
& \hat{\mathbf{u}}_{1}=\mathbf{L}_{1 / 2} \cdot\left[\hat{\mathbf{u}}+\frac{1}{2} \Delta t \hat{\mathcal{N}}(\hat{\mathbf{u}})\right], \\
& \hat{\mathbf{u}}_{2}=\mathbf{L}_{1 / 2} \cdot \hat{\mathbf{u}}+\frac{1}{2} \Delta t \hat{\mathcal{N}}_{1}, \\
& \hat{\mathbf{u}}_{3}=\mathbf{L} \cdot \hat{\mathbf{u}}+\Delta t \mathbf{L}_{1 / 2} \cdot \hat{\mathcal{N}}_{2},
\end{aligned}
$$

with $\hat{\mathcal{N}}_{j}=\hat{\mathcal{N}}\left(\hat{\mathbf{u}}_{j}\right)$.

The fast-inhibitor limit. In the limit $\epsilon=0$, Eq. (2.17), the single equation of motion for $u$ contains a contribution that while nonlocal is nevertheless linear. Since it is a convolution, it is local in Fourier space and can be incorporated directly into the linear operator $\mathcal{L}$. The re- sulting transform is

$$
\omega(k)=-D k^{2}-r+\rho \frac{k^{2}}{1+k^{2}},
$$

precisely the growth rate of the mode + in Eq. (2.7). The diffusive contribution $-D k^{2}$ dominates at large wave vector.

Contour dynamics. In numerical studies of the contour dynamics, we have employed techniques described elsewhere $[39,43]$, summarized briefly here. Starting from an equation of motion in the form

$$
\mathbf{r}_{t}=U \hat{\mathbf{n}}+W \hat{\mathbf{t}}
$$

we study the evolution of the tangent angle [49] $\theta(s)$, related to the curvature by $\kappa(s)=\partial \theta / \partial s[49]$,

$$
\frac{\partial \theta}{\partial t}=-\frac{\partial U}{\partial s}+\kappa W \text {. }
$$

The spectral method described above requires that we utilize a periodic function. A convenient choice is the deviation $\psi$ of $\theta$ from the linear form $\theta=2 \pi s / L$ for a circle,

$$
\theta(\alpha, t)=2 \pi \alpha+\psi(\alpha, t) .
$$

A natural choice of gauge is that of "relative arclength," for which equally spaced points in the parametrization $\alpha=s / L$ remain equally spaced in time. This corresponds to a tangential velocity $[39,43,49]$

$$
W(\alpha)=L\left(\alpha \int_{0}^{1} d \alpha^{\prime} \kappa U-\int_{0}^{\alpha} d \alpha^{\prime} \kappa U\right) .
$$

The highest-order arclength derivative in the $\psi$ dynamics is then diffusive,

$$
\frac{\partial \psi}{\partial t}=\frac{\gamma}{L^{2}} \frac{\partial^{2} \psi}{\partial \alpha^{2}}+\cdots
$$

and amenable to the integrating factor method outlined above. The dynamical variables of the problem are then $\psi$ and the contour length $L$ which obeys the simple evolution

$$
L_{t}=\oint d s \kappa U
$$

[1] K.J. Lee, W.D. McCormick, Q. Ouyang, and H.L. Swinney, Science 261, 192 (1993).

[2] K.J. Lee and H.L. Swinney, Phys. Rev. E 51, 1899 (1995).

[3] A.M. Turing, Philos. Trans. R. Soc. London Ser. B 237, 37 (1952).

[4] V. Castets, E. Dulos, J. Boissonade, and P. De Kepper, Phys. Rev. Lett. 64, 2953 (1990).

[5] Q. Ouyang and H.L. Swinney, Nature 352, 610 (1991).

[6] For a review of recent experiments on the Turing instability, see I. Lengyel and I.R. Epstein, Acc. Chem. Res. 26, 235 (1993).

[7] S. Koga and Y. Kuramoto, Prog. Theor. Phys. 63, 106
(1980).

[8] T. Ohta, M. Mimura, and R. Kobayashi, Physica D 34, 115 (1989).

[9] Localized states may also under oscillatory instabilities [8]. The interplay between these and the steady fingering instabilities has been considered by P. Hirschberg, V. Kirk, and E. Knobloch, Phys. Lett. A 172, 141 (1992).

[10] D.M. Petrich and R.E. Goldstein, Phys. Rev. Lett. 72, 1120 (1994).

[11] R. FitzHugh, Biophys. J. 1, 445 (1961); J.S. Nagumo, S. Arimoto, and Y. Yoshizawa, Proc. IRE 50, 2061 (1962); R. FitzHugh, in Biological Engineering, edited by H.P. 
Schwan (McGraw-Hill, New York, 1969).

[12] J.D. Murray, Mathematical Biology (Springer-Verlag, New York, 1989).

[13] J. Rubinstein, P. Sternberg, and J. Keller, SIAM J. Appl. Math. 49, 116 (1989).

[14] A. Gierer and H. Meinhardt, in Lectures on Mathematics in the Life Sciences (American Mathematical Society, Providence, RI, 1974), Vol. 7, p. 163; H. Meinhardt, Differentiation 6, 117 (1976).

[15] G.B. Ermentrout, S.P. Hastings, and W.C. Troy, SIAM J. Appl. Math 44, 1133 (1984).

[16] For reviews, see H. Möhwald, Annu. Rev. Phys. Chem. 41, 441 (1990); H.M. McConnell, ibid. 42, 171 (1991).

[17] R.E. Rosensweig, Ferrohydrodynamics (Cambridge University Press, Cambridge, 1985).

[18] A.O. Tsebers and M.M. Mairov, Magnetohydrodynamics 16, 21 (1980).

[19] A.G. Boudouvis, J.L. Puchalla, and L.E. Scriven, J. Colloid Interface Sci. 124, 688 (1988).

[20] A.J. Dickstein, S. Erramilli, R.E. Goldstein, D.P. Jackson, and S.A. Langer, Science 261, 1012 (1993).

[21] R.P. Huebener, Magnetic Flux Structures in Superconductors (Springer-Verlag, New York, 1979).

[22] M. Seul, L.R. Monar, L. O'Gorman, and R. Wolfe, Science 254, 1616 (1991).

[23] M. Seul and D. Andelman, Science 267, 476 (1995).

[24] P. Gray and S.K. Scott, Chem. Eng. Sci. 38, 29 (1983); 39, 1087 (1984); J. Phys. Chem. 89, 22 (1985).

[25] J.E. Pearson, Science 261, 189 (1993).

[26] A. Hagberg and E. Meron, Phys. Rev. Lett. 72, 2494 (1994); Nonlinearity 7, 805 (1994).

[27] A. Hagberg and E. Meron, Chaos 4, 477 (1994); C. Elphick, A. Hagberg, and E. Meron, Phys. Rev. E 51, 3052 (1995).

[28] K.J. Lee, W.D. McCormick, J.E. Pearson, and H.L. Swinney, Nature 214, 215 (1994).

[29] W.N. Reynolds, J.E. Pearson, and S. Ponce-Dawson, Phys. Rev. Lett. 72, 2797 (1994).

[30] K. Krischer and A. Mikhailov, Phys. Rev. Lett. 73, 3165 (1994).

[31] See, e.g. P.C. Fife, Dynamics of Internal Layers and Diffusive Interfaces (SIAM, Philadelphia, 1988), and references therein.

[32] J.P. Keener, SIAM J. Appl. Math. 46, 1039 (1986); A.T. Winfree, SIAM Rev. 32, 1 (1990); A.J. Bernoff, Physica D 53, 125 (1991); E. Meron, Phys. Rep. 218, 1 (1992), and references therein.

[33] We are grateful to A.J. Bernoff (private communication, 1993) for discussions concerning this point.

[34] T. Ohta, A. Ito, and A. Tetsuka, Phys. Rev. A 42, 3225 (1990).

[35] J.B. Swift and P.C. Hohenberg, Phys. Rev. A 15, 319 (1977).

[36] R.M. Hornreich, M. Luban, and S. Shtrikman, Phys. Rev. Lett. 35, 1678 (1975).

[37] H. Goldstein, Classical Mechanics (Addison-Wesley, Reading, PA, 1980), p. 24.

[38] A.T. Dorsey, Ann. Phys. 233, 248 (1994).

[39] S.A. Langer, R.E. Goldstein, and D.P. Jackson, Phys. Rev. A 46, 4894 (1992).

[40] G.F. Carrier, M. Krook, and C.E. Pearson, Functions of a Complex Variable (McGraw-Hill, New York, 1966).

[41] W.W. Mullins and R.F. Sekerka, J. Appl. Phys. 35, 444
(1964).

[42] D.P. Jackson, R.E. Goldstein, and A.O. Cebers, Phys. Rev. E 50, 298 (1994).

[43] R.E. Goldstein and D.P. Jackson, J. Phys. Chem. 98, 9626 (1994).

[44] B. Duplantier, R.E. Goldstein, V. Romero-Rochín, and A.I. Pesci, Phys. Rev. Lett. 65, 508 (1990).

[45] E.J.W. Verwey and J.Th.G. Overbeek, Theory of the Stability of Lyophobic Colloids (Elsevier, Amsterdam, 1948).

[46] L.D. Landau and E.M. Lifshitz, Theory of Elasticity (Pergamon Press, New York, 1959), pp. 17 and 18.

[47] J. Langer and D.A. Singer, Topology 24, 75 (1985).

[48] R.E. Goldstein and S.A. Langer, Phys. Rev. Lett. 75, 1094 (1995).

[49] R.C. Brower, D.A. Kessler, J. Koplik, and H. Levine, Phys. Rev. A 29, 1335 (1984).

[50] See, for instance, the discussion of Steiner trees in C. Isenberg, The Science of Soap Films and Soap Bubbles (Dover, New York, 1992), Chap. 3.

[51] J.S. Langer, Acta Metall. 25, 1121 (1977).

[52] R.E. Goldstein (unpublished).

[53] M. Doi and S.F. Edwards, The Theory of Polymer Dynamics (Oxford University Press, New York, 1986).

[54] D.J. Muraki and R.E. Goldstein (unpublished).

[55] R.E. Goldstein, D.P. Jackson, and A.T. Dorsey (unpublished).

[56] A.O. Cebers, Magnetohydrodynamics 25, 149 (1989).

[57] D.J. Keller, J.P. Korb, and H.M. McConnell, J. Phys. Chem. 91, 6417 (1987).

[58] J. Pearl, Appl. Phys. Lett. 5, 65 (1964).

[59] A.L. Fetter and P.C. Hohenberg, Phys. Rev. 159, 330 (1967).

[60] A.B. Pippard, Philos. Mag. 41, 243 (1950).

[61] K.Y.C. Lee and H.M. McConnell, J. Phys. Chem. 97, 9532 (1993).

[62] H.A. Stone and H.M. McConnell, Proc. R. Soc. London Ser. A 448, 97 (1995).

[63] D.K. Lubensky and R.E. Goldstein, Phys. Fluids (to be published).

[64] T. Ohta and K. Kawasaki, Macromolecules 19, 2621 (1986).

[65] J. Weeks, private communication.

[66] E. Ben-Jacob, N. Goldenfeld, J.S. Langer, and G. Schön, Phys. Rev. A 29, 330 (1984).

[67] R.E. Goldstein and D.M. Petrich, Phys. Rev. Lett. 69, 555 (1992).

[68] N.J. Zabusky, M.H. Hughes, and K.V. Roberts, J. Comput. Phys. 30, 96 (1976).

[69] D.G. Dritschel, J. Fluid Mech. 172, 157 (1986).

[70] E.C. Edblom, M. Györgyi, M. Orbán, and I.R. Epstein, J. Am. Chem. Soc. 108, 2826 (1986).

[71] V. Gáspár and K. Showalter, J. Phys. Chem. 94, 4973 (1990), and references therein.

[72] See, e.g., Spectral Methods in Fluid Mechanics, edited by C. Canuto, M.Y. Hussaini, A. Quarteroni, and T.A. Zang (Springer-Verlag, New York, 1988), p. 112; R.S. Rogallo, NASA Report No. TM-73203 (1977); P.R. Spalart, NASA Report No. TM-88222 (1986).

[73] We are indebted to M.J. Shelley for valuable discussions regarding these numerical methods.

[74] W.H. Press, S.A. Teukolsky, W.T. Vetterling, and B.P. Flannery, Numerical Recipes in $C$, 2nd ed. (Cambridge University Press, Cambridge, 1992). 